\title{
Universal Vector-Scalar Potential Framework for Inhomogeneous Electromagnetic System and Its Application in Semiclassical Quantum Electromagnetics
}

\author{
Guoda Xie ${ }^{\circledR}$, Zhixiang Huang ${ }^{\circledR}$, Senior Member, IEEE, Jian Wei You ${ }^{\circledR}$, Senior Member, IEEE, Zhihao Lan ${ }^{(\mathbb{}}$, \\ Nicolae C. Panoiu ${ }^{\circledR}$, Member, IEEE, and Wei E. I. Sha ${ }^{\circledR}$, Senior Member, IEEE
}

\begin{abstract}
In this work, numerical solution to a general electromagnetic (EM) system is studied using a formalism based on the formulas for the $\mathrm{E}-\mathrm{B}-\mathrm{A}-\phi$ formulas with different gauge conditions. The finite-difference time-domain (FDTD) method is employed to discretize these formulas. In addition, the convolutional perfectly matched layer (CPML) technique is successfully applied to absorb outgoing scattered waves described by the proposed formulas. The gauge invariance of EM fields in inhomogeneous environment is demonstrated by numerical examples. Moreover, the proposed EM framework integrated with the Schrödinger equation is introduced to investigate the mesoscopic phenomenon for light-matter interaction, which is useful to design laser pulses for controlling discrete quantum states. The work offers a simple and general numerical EM framework, which is essential to bridge the classical EM and quantum mechanical systems.
\end{abstract}

Manuscript received May 18, 2021; revised August 16, 2021; accepted September 23, 2021. This work was supported in part by the National Science Foundation of China (NSFC) under Grant U20A20164, Grant 61975177, Grant 61971001, Grant 61871001, Grant 62171001, and Grant 61701003; in part by the National Science Foundation (NSF) of Anhui Province under Grant 1908085QF257, Grant 2108085MF198, Grant KJ2018A0015, and Grant K120436040; in part by the Open Fund for Discipline Construction, Institute of Physical Science and Information Technology, Anhui University, under Grant 2019AH001; and in part by the Marie Sklodowska-Curie Individual Fellowship under Grant MSCA-IF-EF-ST (752898). The work of Jian Wei You, Zhihao Lan, and Nicolae C. Panoiu was supported by European Research Council (ERC) under Grant ERC-2014-CoG-648328. The review of this article was arranged by Senior Editor F. Taccogna. (Corresponding author: Wei E. I. Sha.)

Guoda Xie is with the Key Laboratory of Intelligent Computing and Signal Processing, Ministry of Education, and the Key Laboratory of Electromagnetic Environmental Sensing, Department of Education of Anhui, Anhui University, Hefei 230601, China, and also with the Key Laboratory of Micro-Nano Electronic Devices and Smart Systems of Zhejiang, College of Information Science and Electronic Engineering, Zhejiang University, Hangzhou 310027, China.

Zhixiang Huang is with the Key Laboratory of Intelligent Computing and Signal Processing, Ministry of Education, and the Key Laboratory of Electromagnetic Environmental Sensing, Department of Education of Anhui, Anhui University, Hefei 230601, China (e-mail: zxhuang@ahu.edu.cn).

Jian Wei You, Zhihao Lan, and Nicolae C. Panoiu are with the Department of Electronic and Electrical Engineering, University College London, London WC1E 7JE, U.K. (e-mail: n.panoiu@ucl.ac.uk).

Wei E. I. Sha is with the Key Laboratory of Micro-Nano Electronic Devices and Smart Systems of Zhejiang, College of Information Science and Electronic Engineering, Zhejiang University, Hangzhou 310027, China (e-mail: weisha@zju.edu.cn).

Color versions of one or more figures in this article are available at https://doi.org/10.1109/TPS.2021.3117607.

Digital Object Identifier 10.1109/TPS.2021.3117607
Index Terms-Convolutional perfectly matched layer (CPML), electromagnetic (EM) system, finite-difference time-domain (FDTD), gauge invariance, vector potential and scalar potential formulas.

\section{INTRODUCTION}

O OMPUTATIONAL electromagnetics (CEM) [1] solves scientific and engineering problems in the EM regime, such as antenna design and optimization [2], ground penetrating radar [3], and electronic devices [4]. When the size of highly integrated devices approaches the nanoscale, several quantum effects, such as spill-out and tunneling effects of electrons [5], and electron transition [6] of atoms and molecules, become increasingly more significant in the EM system and cannot be ignored. In these cases, the classical EM framework is no longer applicable, and these quantum effects will lead to a crucial influence on the performance of photonic and electronic nanodevices. Moreover, these quantum effects can be applied to improve device performance and enhance their functionality. Therefore, the study of the interaction between artificial atoms and external EM fields has become an important and rapidly growing research topic in developing next-generation electronic technologies. Equally important, designing nanodevices and understanding their quantum properties via practical measurements can be extremely expensive and time-consuming. Therefore, the development of an efficient numerical simulation platform for EM response of a nanoscale system is desirable. In fact, some numerical methods based on Maxwell-Schrödinger (M-S) equations [7]-[10] or Maxwell-Bloch equations [11]-[13] have been proposed to model the multiphysics and multiscale problems in a hybrid regime of classical EM and quantum worlds.

In order to bridge the quantum mechanics (QM) and classical EM, an alternative form of Maxwell equations based on the vector potential (A) and scalar potential $(\phi)$ formulas is proposed, instead of employing traditional $\mathbf{H}$ fields [14], [15]. However, the second-order partial differential operators acting on the field components in temporal and spatial domains make the numerical implementation of the formulas difficult, especially for the perfectly matched layer (PML) technique 
used to truncate the computational domain and absorb outwardly propagating waves. To investigate the dynamics of charge wave packets in a carbon nanotube, a hybrid method utilizing the transmission line matrix [16] method and finitedifference time-domain (FDTD) approach [17] was employed to solve for the $\mathrm{M}-\mathrm{S}$ equation. In addition, other hybrid numerical methods, such as the locally one-dimensional (LOD) FDTD method [18] and alternating-direction-implicit (ADI) FDTD method, were also used to solve for the coupled M-S equations for simulating nanodevices [19]. Moreover, based on the solution to the $\mathrm{M}-\mathrm{S}$ equations, some other multiphysics simulations, such as the modeling of optically controlled quantum states with the Lorenz gauge [20] and the modeling of EM field-particle interaction with the Coulomb gauge [21], [22], were also developed. However, the discussion of the gauge invariance is not comprehensive in the abovementioned works. In most of these cases, one simply chose a suitable type of field gauge for a specific problem.

In this article, we propose a simple but general $\mathbf{E}-\mathbf{B}-\mathbf{A}-\phi$ formulas that necessitate first-order partial differential operators both in time and space domains. The proposed numerical framework can be used to simulate the EM response of inhomogeneous and dispersive media. In particular, the Lorenz gauge with different forms is applied in the proposed framework to demonstrate the gauge invariance of EM fields. In addition, the convolutional PML (CPML) technique is successfully applied to the coupled EM system described by the proposed formulas. Meanwhile, the accuracy of the numerical framework established and the gauge invariance of EM fields are verified by several numerical examples. Finally, the proposed hybrid EM system coupled to the Schrödinger equation is employed to simulate the transient interaction between an EM control pulse and a single electron that is confined in a quasi-one-dimensional nanoscale potential well. The simulation results show that the proposed method could reproduce a perfect quantum state switching while with a more straightforward numerical solution process. This article is organized as follows. In Section II, the decoupled $\mathbf{A}$ and $\phi$ equations and the proposed $\mathbf{E}-\mathbf{B}-\mathbf{A}-\phi$ formulas are presented, and the iteration procedures and the spatial distribution of the field components are introduced. Then, the method of applying the CPML technique to the proposed EM system is introduced. In Section III, several numerical examples are adopted to verify both the accuracy of the proposed method and the gauge invariance of the EM fields. In Section IV, the combination of the proposed EM system with the Schrödinger equation is presented to design the laser pulse for controlling discrete quantum states. Finally, the conclusion and outlook are presented in Section V.

\section{EM System With Vector and Scaler Potentials}

\section{A. $E-B-A-\phi$ Formulas of EM System}

The time-harmonic form of Maxwell's equations in linear, isotropic, and nonmagnetic medium can be written as

$$
\begin{aligned}
-j \omega \mathbf{B}(\mathbf{r}) & =\nabla \times \mathbf{E}(\mathbf{r}) \\
j \omega \mathbf{D}(\mathbf{r})+\mathbf{J}(\mathbf{r}) & =\nabla \times \mathbf{H}(\mathbf{r})
\end{aligned}
$$

$$
\begin{aligned}
& \nabla \cdot \mathbf{B}(\mathbf{r})=0 \\
& \nabla \cdot \mathbf{D}(\mathbf{r})=\rho(\mathbf{r}) .
\end{aligned}
$$

The constitutive relations between these field components are to be described by

$$
\mathbf{D}(\mathbf{r}, \omega)=\varepsilon(\mathbf{r}, \omega) \mathbf{E}(\mathbf{r}, \omega), \mathbf{B}(\mathbf{r}, \omega)=\mu \mathbf{H}(\mathbf{r}, \omega)
$$

where $\varepsilon(\mathbf{r}, \omega)$ is the inhomogeneous permittivity of the dispersive medium and $\mu$ is the constant permeability.

According to the above constitutive relations, (2) is rewritten as

$$
j \omega \varepsilon(\mathbf{r}) \mathbf{E}(\mathbf{r})+\mathbf{J}(\mathbf{r})=\mu^{-1} \nabla \times \mathbf{B}(\mathbf{r}) .
$$

By using the divergence-free condition $\nabla \cdot \mathbf{B}=0$, one can introduce the vector potential A through

$$
\mathbf{B}(\mathbf{r})=\nabla \times \mathbf{A}(\mathbf{r})
$$

Using (1), the relation among $\mathbf{A}, \phi$, and $\mathbf{E}$ satisfies the following:

$$
j \omega \mathbf{A}(\mathbf{r})=-\mathbf{E}(\mathbf{r})-\nabla \phi(\mathbf{r}) .
$$

Substituting (8) into (4), we have

$$
j \omega \nabla \cdot(\varepsilon(\mathbf{r}) \mathbf{A}(\mathbf{r}))+\nabla \cdot(\varepsilon(\mathbf{r}) \nabla \phi(\mathbf{r}))=-\rho(\mathbf{r}) .
$$

Similarly, substituting (7) and (8) into (2), then

$$
\mu^{-1} \nabla \times \nabla \times \mathbf{A}(\mathbf{r})+(j \omega)^{2} \varepsilon(\mathbf{r}) \mathbf{A}(\mathbf{r})+j \omega \varepsilon(\mathbf{r}) \nabla \phi(\mathbf{r})=\mathbf{J}(\mathbf{r}) .
$$

To derive the decoupled $\mathbf{A}-\phi$ formulas, one method is to use the simple Lorenz gauge

$$
\nabla \cdot \mathbf{A}(\mathbf{r})=-\mu \varepsilon j \omega \phi(\mathbf{r}) .
$$

It is easy to prove using (11) that the coupled $\mathbf{A}-\phi$ formulas can be decoupled in homogeneous media $(\varepsilon(\mathbf{r})=\varepsilon)$

$$
\begin{aligned}
\nabla^{2} \phi-\mu \varepsilon(j \omega)^{2} \phi & =-\rho / \varepsilon \\
\nabla^{2} \mathbf{A}-\mu \varepsilon(j \omega)^{2} \mathbf{A} & =-\mu \mathbf{J} .
\end{aligned}
$$

Another gauge is the generalized Lorenz gauge [23]

$$
\varepsilon(\mathbf{r})^{-1} \nabla \cdot(\varepsilon(\mathbf{r}) \mathbf{A}(\mathbf{r}))=-\mu \varepsilon(\mathbf{r}) j \omega \phi(\mathbf{r}) .
$$

The generalized Lorenz gauge defined by (14) can lead to the decoupled $\mathbf{A}-\phi$ formulas in inhomogeneous media [14], i.e.,

$$
\begin{gathered}
\nabla \cdot(\varepsilon(\mathbf{r}) \nabla \phi(\mathbf{r}))-\boldsymbol{\mu} \varepsilon(\mathbf{r})^{2}(j \omega)^{2} \phi(\mathbf{r})=-\rho \\
-\mathbf{J}=-\mu^{-1} \nabla \times \nabla \times \mathbf{A}(\mathbf{r})-(j \omega)^{2} \varepsilon(\mathbf{r}) \mathbf{A}(\mathbf{r}) \\
+\mu^{-1} \varepsilon(\mathbf{r}) \nabla\left[\varepsilon(\mathbf{r})^{-2} \nabla \cdot(\varepsilon(\mathbf{r}) \mathbf{A}(\mathbf{r}))\right] .
\end{gathered}
$$

The two sources in the $\mathbf{A}-\phi$ formulas are related to the current continuity equation $\nabla \cdot \mathbf{J}+j \omega \rho=0$. In order to get the solution of the decoupled $\mathbf{A}-\phi$ formulas (12) and (13) or (15) and (16), one needs to numerically solve the secondorder partial differential equations (PDEs), which is usually time-consuming and complicated when the FDTD method is applied in time domain.

Instead of directly solving the formulas (12) and (13) or (15) and (16), a novel iteration approach based on the 


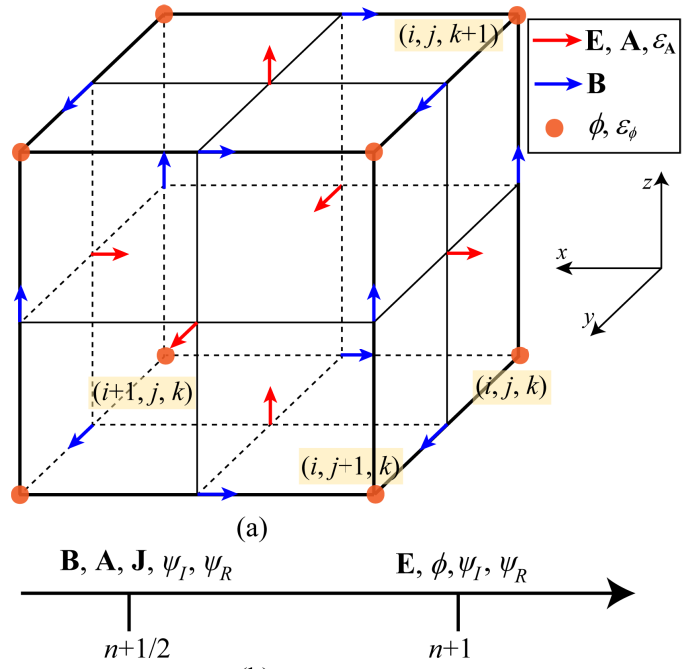

(b)

Fig. 1. Space and time configurations of the field components $(\mathbf{E}, \mathbf{B}, \mathbf{A}, \mathbf{J}$ $\phi, \psi_{R}$, and $\left.\psi_{I}\right)$ on a Yee grid indexed as $(i, j$, and $k)$ and $n+1 / 2$ and $n+1$ indicate the points on the time axis.

$\mathbf{E}-\mathbf{B}-\mathbf{A}-\phi$ formulas is proposed to simulate a series of firstorder PDEs in sequence. As a result, the $\mathbf{E}-\mathbf{B}-\mathbf{A}-\phi$ formulas can be implemented much more efficiently, particularly for the implementation of PML. Moreover, the gauge condition (11) or (14) can be conveniently adopted to prove the gauge invariance as well. According to the relation between EM field components and potential field components, (6)-(8) can be coupled via the Lorenz gauge condition (11) or (14) to form a self-consistent numerical system, where only first-order PDEs are to be discretized.

To numerically solve the self-consistent system, i.e., $(6-8,11)$ or $(6-8,14)$, we can transform the time-harmonic form of the $\mathbf{E}-\mathbf{B}-\mathbf{A}-\phi$ formulas into corresponding timedomain form, which could be easily simulated with the FDTD method. It is shown that the complexity of the proposed $\mathbf{E}-\mathbf{B}-\mathbf{A}-\phi$ formulas is about 1.5 times larger than that of the traditional $\mathbf{E}-\mathbf{H}$ curl equations.

In the Yee grid of the FDTD method, the electric fields are located at the centers of its edges, and magnetic fields are located at the centers of its faces, as shown in Fig. 1. In our $\mathbf{E}-\mathbf{B}-\mathbf{A}-\phi$ framework, $\mathbf{A}$ and $\mathbf{E}$ are placed at the same positions. According to the Lorenz gauge, the positions of A and $\phi$ are half a grid apart, and thus, $\phi$ is located at the corner of the Yee cell. It must be noted that, for the generalized Lorenz gauge, the location of permittivity $\varepsilon(\mathbf{r})$ on the left-hand side of (14) should be the same as that of A. Similarly, the location of the permittivity $\varepsilon(\mathbf{r})$ on the right-hand side of (14) should be the same as that of $\phi$. The discrete spatial positions of these field components in a Yee cell are shown in Fig. 1.

The time-evolution procedure of the $\mathbf{E}-\mathbf{B}-\mathbf{A}-\phi$ framework is shown in Fig. 2. First, one sets the simulation domain and defines the object in space. Subsequently, in order to reduce the simulation time, some time-independent coefficients are evaluated before starting the time-evaluation loop. At each iteration step, the $\mathbf{B}$ components are calculated by using the value of $\mathbf{A}$, and then, the value of $\mathbf{B}$ is used to update $\mathbf{E}$.

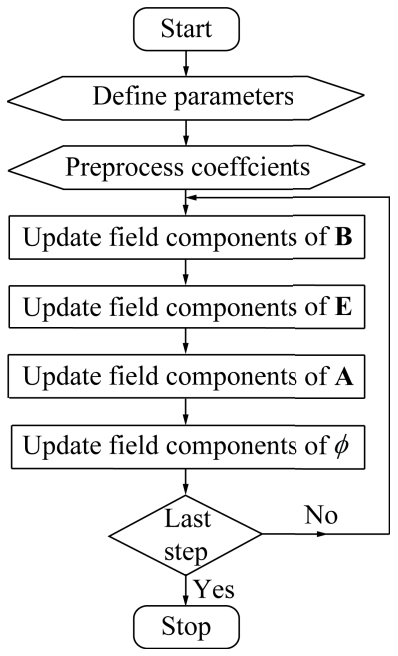

Fig. 2. Schematic of the numerical method for updating the field components in the $\mathbf{E}-\mathbf{B}-\mathbf{A}-\phi$ system.

Using the values of $\mathbf{E}$ and $\phi$, the components of $\mathbf{A}$ are evaluated, and then, the corresponding results are used to update the value of $\phi$. Simultaneously, the updating equations of the proposed $\mathbf{E}-\mathbf{B}-\mathbf{A}-\phi$ framework are described in detail in the Appendix.

\section{B. CPML for the Proposed EM System}

In addition, to numerically simulate the wave propagation in infinite space, the CPML technique is adopted in the $\mathbf{E}-\mathbf{B}-\mathbf{A}-\phi$ system. According to the CPML technique, the spatial differential operator $\nabla$ should be modified as

$$
\nabla_{s}=\frac{1}{s_{x}} \frac{\partial}{\partial x} \mathbf{e}_{x}+\frac{1}{s_{y}} \frac{\partial}{\partial y} \mathbf{e}_{y}+\frac{1}{s_{z}} \frac{\partial}{\partial z} \mathbf{e}_{z}
$$

where

$$
s_{u}=k_{u}+\frac{\sigma_{u}}{\alpha_{u}+j \omega \varepsilon_{0}} .
$$

The quantity $s_{u}$ is a coordinate stretched factor, and the subscript $u=x, y$, and $z$. The parameters in the CPML technique are scaling factors with a polynomial profile $m$, and the specific expressions of these parameters are given in [24].

Using a recursive convolution method, the updating equations of the field components along the $x$-direction take the following form

$$
\begin{aligned}
\varepsilon \frac{\partial E_{x}}{\partial t} & =\frac{1}{k_{y}} \frac{\partial B_{z}}{\partial y}-\frac{1}{k_{z}} \frac{\partial B_{y}}{\partial z}+\Psi_{E x y}-\Psi_{E x z} \\
B_{x} & =\frac{1}{k_{y}} \frac{\partial A_{z}}{\partial y}-\frac{1}{k_{z}} \frac{\partial A_{y}}{\partial z}+\Psi_{B x y}-\Psi_{B x z} \\
\frac{\partial A_{x}}{\partial t} & =-\frac{1}{k_{x}} \frac{\partial \phi}{\partial x}-\Psi_{A x x}-E_{x} .
\end{aligned}
$$

The formula of the generalized Lorenz gauge in the CPML region can be derived as

$$
\begin{array}{r}
\frac{\partial \phi}{\partial t}=-\frac{1}{\mu \varepsilon_{\phi}^{2}}\left(\frac{1}{k_{x}} \frac{\partial\left(\varepsilon_{x A} A_{x}\right)}{\partial x}+\frac{1}{k_{y}} \frac{\partial\left(\varepsilon_{y A} A_{y}\right)}{\partial y}+\frac{1}{k_{z}} \frac{\partial\left(\varepsilon_{z A} A_{z}\right)}{\partial z}\right) \\
+\Psi_{\phi x}+\Psi_{\phi y}+\Psi_{\phi z} . \quad(22)
\end{array}
$$




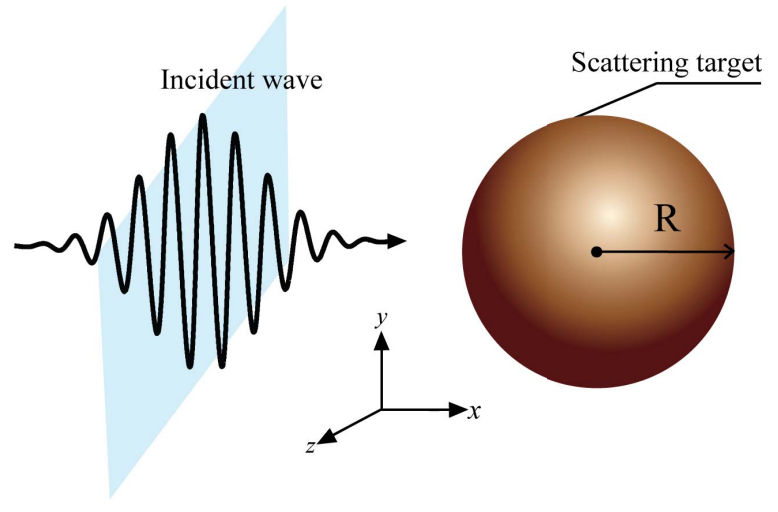

Fig. 3. Dielectric sphere illuminated by an EM plane wave.

The auxiliary variables $\Psi$ in (19)-(22) can be updated by using the corresponding field components. For instance, the auxiliary variable in (22) along the $x$-direction takes the forms

$$
\Psi_{\phi x_{i, j, k}}^{n+1}=b_{x} \Psi_{\phi x_{i, j, k}}^{n}+c_{x} \frac{\partial\left(\varepsilon_{x A} A_{x}\right)}{\partial x}
$$

and

$$
\begin{aligned}
b_{x} & =e^{-\left(\left(\sigma_{x} / k_{x}\right)+\alpha_{x}\right)\left(\Delta t / \varepsilon_{0}\right)} \\
c_{x} & =\frac{\sigma_{x}}{\sigma_{x} k_{x}+\alpha_{x} k_{x}^{2}}\left(e^{-\left(\left(\sigma_{x} / k_{x}\right)+\alpha_{x}\right)\left(\Delta t / \varepsilon_{0}\right)}-1\right) .
\end{aligned}
$$

It is worth noting that the proposed $\mathbf{E}-\mathbf{B}-\mathbf{A}-\phi$ numerical system should be divided into two parts when operating the CPML parameters: one is the $\mathbf{E}-\mathbf{B}$ and the other is the $\mathbf{A}-\phi$. The settings of the CPML parameters for the field components $\mathbf{E}-\mathbf{B}$ are the same as those of the field components $\mathbf{E}-\mathbf{H}$. It is known that the CPML parameters for field components $\mathbf{E}-\mathbf{H}$ are defined by their locations in space domain. It can be seen from Fig. 1 that the field components $\mathbf{E}$ are located at the ends of the edges and the field components $\mathbf{B}$ are located at the centers of the edges. Therefore, the distance of half a grid between $\mathbf{E}$ and $\mathbf{B}$ in each direction should be considered when defining the CPML parameters. Although A and $\mathbf{E}$ are placed at the same positions, however, the field components $\mathbf{A}$ are located at the centers of the edges and the field components $\phi$ are located at the ends of the edges in each direction when considering the $\mathbf{A}-\phi$ part. Through the above analyses, it is known that the settings of CPML parameters for the field components $\mathbf{A}$ should be the same as those of the field components B. Similarly, the settings of CPML parameters for the field components $\phi$ should be the same as those of the field components $\mathbf{E}$. The validation and flexibility of the proposed method for the CPML technique applied in this EM system have been investigated in the following section.

\section{NUMERICAL EXAMPLES FOR EM SYSTEM}

The physical system used to verify the accuracy of the proposed numerical method and its gauge invariance is shown in Fig. 3, where a 3-D lossless dielectric sphere with the relative permittivity $\varepsilon_{r}=4$ is illuminated by a plane-wave excitation. The radius of the sphere is $R=300 \mathrm{~nm}$. The Yee grid size is chosen as $\Delta x=\Delta y=\Delta z=\Delta=15 \mathrm{~nm}$, and

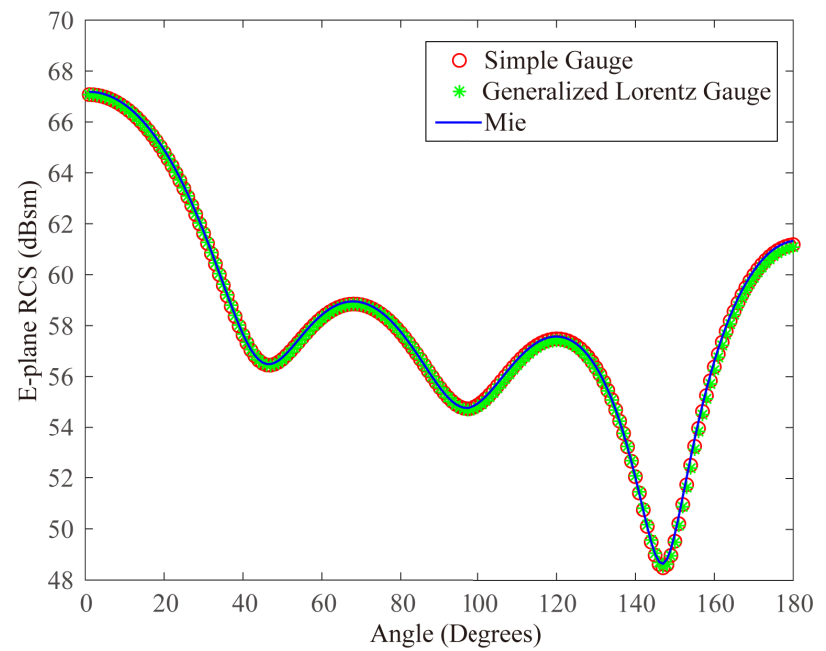

Fig. 4. E-plane bistatic RCS for a lossless dielectric sphere with the size of half wavelength and relative permittivity of 4 .

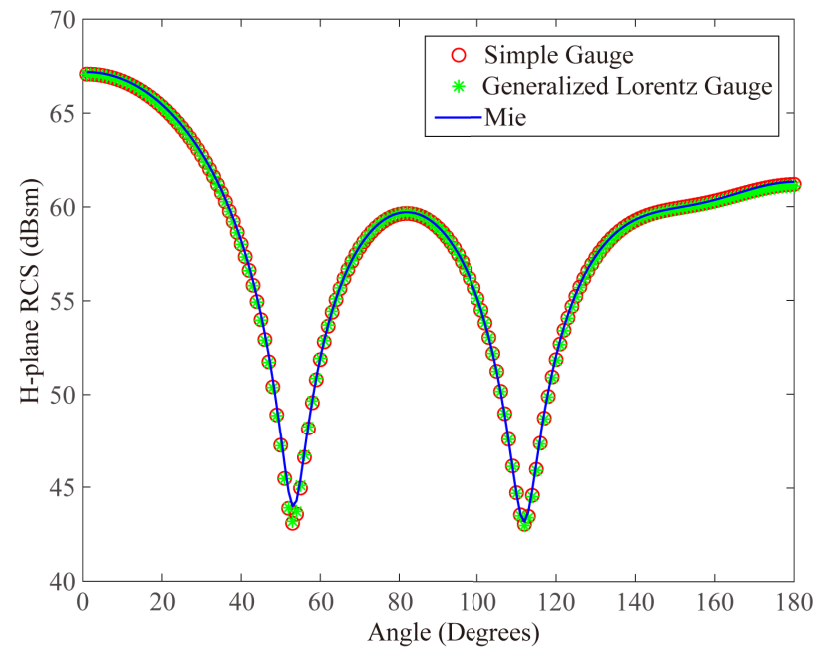

Fig. 5. H-plane bistatic RCS for a lossless dielectric sphere with the size of half wavelength and relative permittivity of 4 .

the time step interval equals 0.78 times the CFL maximum allowed time step in the FDTD method. The computational domain is surrounded by 15 layers of CPML and consists of $91 \times 91 \times 91$ grid cells. The parameters in the CPML technique are scaling factors with a polynomial profile $m(m=1.9$ in this example). The specific expressions of these parameters are given in [24], and the corresponding optimal values are chosen as $k_{\max }=1$ and $\sigma_{\max }=0.5(m+1) /(150 \pi \Delta)$. The incident plane wave has a modulated Gaussian envelope in time with a carrier frequency of $500 \mathrm{THz}$ (wavelength of $600 \mathrm{~nm}$ ) and $t_{0}=3 \tau, \tau=1.5 \mathrm{fs}\left(t_{0}\right.$ denotes the time reaching peak value of the Gaussian pulse). The proposed $\mathbf{A}-\phi$ framework is applied to evaluate the bistatic E- and H-plane radar cross section (RCS) at the center frequency. As shown in Figs. 4 and 5, the numerical results of simple gauge and generalized Lorenz gauge $\left(\varepsilon=4 \varepsilon_{0}\right)$ agree well with each other. Moreover, all the numerical results exhibit very high accuracy compared to the analytical results of Mie series solution. Hence, the accuracy of the proposed numerical framework is verified and the gauge invariance of the EM fields is numerically demonstrated. 


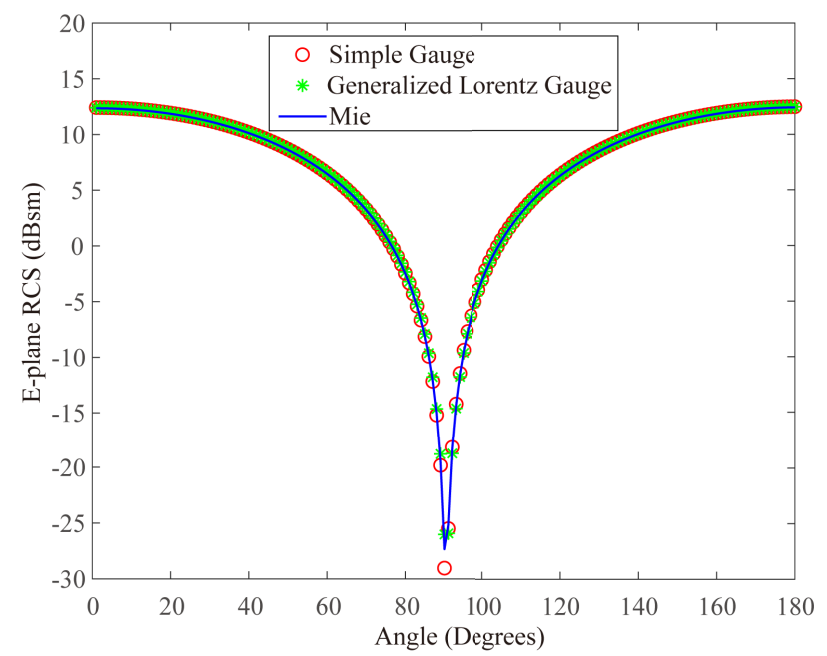

Fig. 6. E-plane bistatic RCS for a dispersive metallic sphere (with the radius of $20 \mathrm{~nm}$ and incident wavelength of $600 \mathrm{~nm}$ ).

To further verify the gauge invariance of the universal $\mathbf{A}-\phi$ formalism, a more complicated 3-D dispersive metallic sphere is studied. The radius of the sphere is $20 \mathrm{~nm}$. The grid size is chosen as $\Delta x=\Delta y=\Delta z=\Delta=1 \mathrm{~nm}$, and the time step interval equals 0.61 times the CFL maximum allowed time step in the FDTD method. In this example, the dispersive permittivity of metallic medium is described by the Drude model as

$$
\varepsilon^{\text {Drude }}(\omega)=\varepsilon_{0}\left[\varepsilon_{\infty}-\chi(\omega)\right]=\varepsilon_{0} \varepsilon_{\infty}-\varepsilon_{0} \frac{\omega_{p}^{2}}{\omega\left(\omega-j \nu_{c}\right)}
$$

where $\varepsilon_{\infty}$ is the relative permittivity at infinitely-large frequencies, $\omega_{p}$ is the plasma frequency, and $v_{c}$ is the damping coefficient. Hence, to simulate dispersive media, (6) can be discretized by the auxiliary differential equation (ADE) scheme [25]. In this example, $\varepsilon_{\infty}=9.5, \omega_{p}=1.36 \times 10^{16} \mathrm{~Hz}$, and $v_{c}=1.048 \times 10^{15} \mathrm{~Hz}$. For simplification, the generalized Lorenz gauge (14) in this example is given by

$$
\left(\varepsilon_{0} \varepsilon_{\infty}\right)^{-1} \nabla \cdot\left(\varepsilon_{0} \varepsilon_{\infty} A\right)=-\mu \varepsilon_{0} \varepsilon_{\infty} j \omega \phi
$$

where $\varepsilon_{\infty}=9.5$ in the sphere region and $\varepsilon_{\infty}=1$ in the air region. Regarding the simple gauge, $\varepsilon_{\infty}=1$ is set for all regions.

The bistatic E-plane RCS of the dispersive metallic sphere is given in Fig. 6, whereas the absorbing cross section (ACS) and scattering cross section (SCS) are presented in Figs. 7 and 8, respectively. These simulation comparisons show that the results obtained by the simple gauge agree well with those obtained by the generalized Lorenz gauge. Moreover, both numerical methods agree well with the analytical results.

Meanwhile, the accuracy of the proposed $\mathbf{E}-\mathbf{B}-\mathbf{A}-\phi$ formulas system in simulating the propagation of the EM field and the absorbing performance of the CPML applied in this system are investigated. Considering the radiation of an electric dipole in a three-dimensional space, the vertical dipole is assumed to be at the center of the computational region. The computational region contains $60 \times 60 \times 60$ Yee cells and the cell sizes are chosen as $\Delta x=\Delta y=\Delta x=0.05 \mathrm{~m}$. A Gaussian

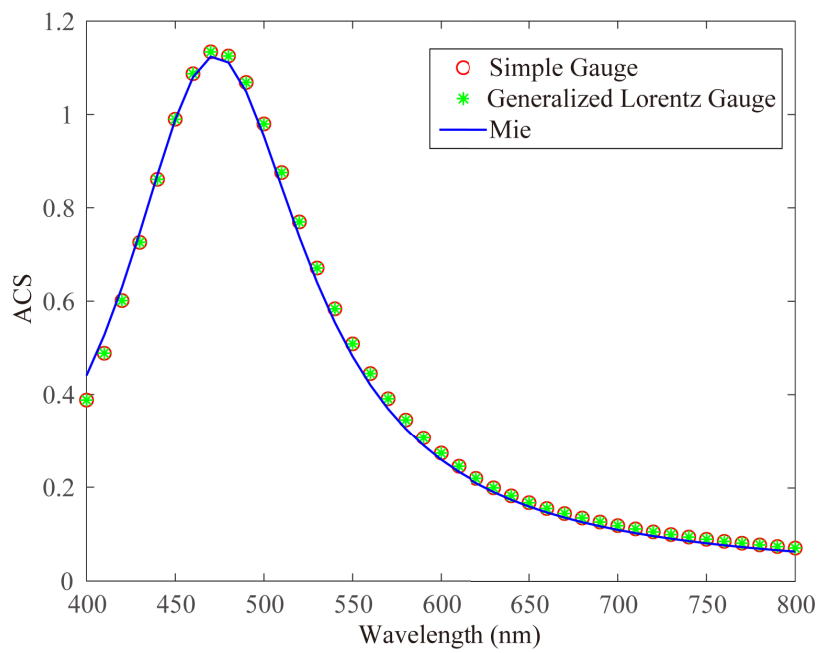

Fig. 7. ACS for a dispersive metallic sphere (with the radius of $20 \mathrm{~nm}$ ).

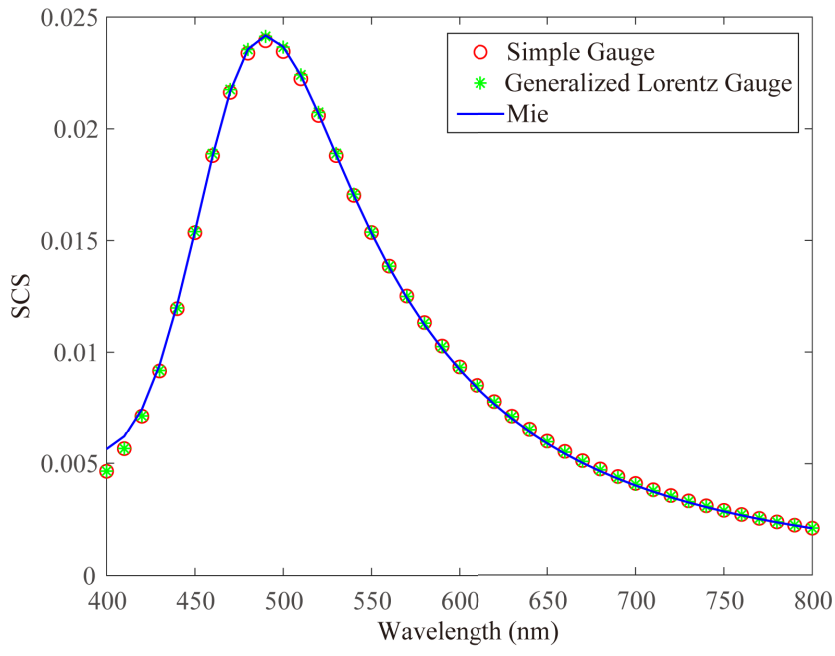

Fig. 8. SCS for a dispersive metallic sphere (with the radius of $20 \mathrm{~nm}$ ).

source with a time-domain form of $e^{((t-3 \tau) / \tau)^{2}}(\tau=2 \mathrm{~ns})$ is used as the radiation source and the probe point is placed two cells away from the interface between the computational domain and the CPML region. The number of the CPML layers is 10 and the parameters are chosen as $m=3$, $\alpha_{\max }=0.04(m+1) /(150 \pi \Delta), \sigma_{\max }=0.5(m+1) /(150 \pi \Delta)$, and $k_{\max }=1$. The radiation results of the electric dipole are shown in Fig. 9. For comparison, the numerical results obtained by using the traditional E-H Maxwell equations (1) and (2), A- $\phi$ framework (12) and (13), and the proposed E-B-A- $\phi$ system are provided. It can be seen that the result obtained by the proposed system has a good agreement with that obtained by the traditional $\mathbf{E}-\mathbf{H}$ and $\mathbf{A}-\phi$ frameworks. Therefore, the accuracy of the proposed system in simulating the propagation of the EM field is demonstrated.

To evaluate the absorbing performance of the CPML applied in this $\mathbf{E}-\mathbf{B}-\mathbf{A}-\phi$ system, the relative reflection errors at the probe point are calculated by the following:

$$
E_{\text {error }}=20 \log _{10} \frac{\left|E(t)-E_{\text {ref }}(t)\right|}{\max \left|\left(E_{\text {ref }}(t)\right)\right|}
$$




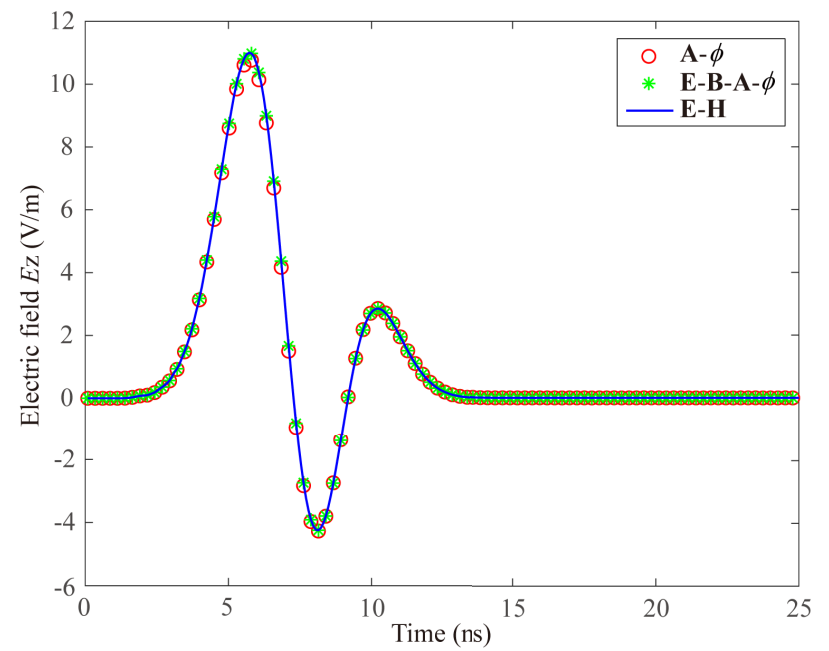

Fig. 9. Radiation field of electric dipole for the $\mathbf{A}-\phi, \mathbf{E}-\mathbf{B}-\mathbf{A}-\phi$, and $\mathbf{E}-\mathbf{H}$ system.

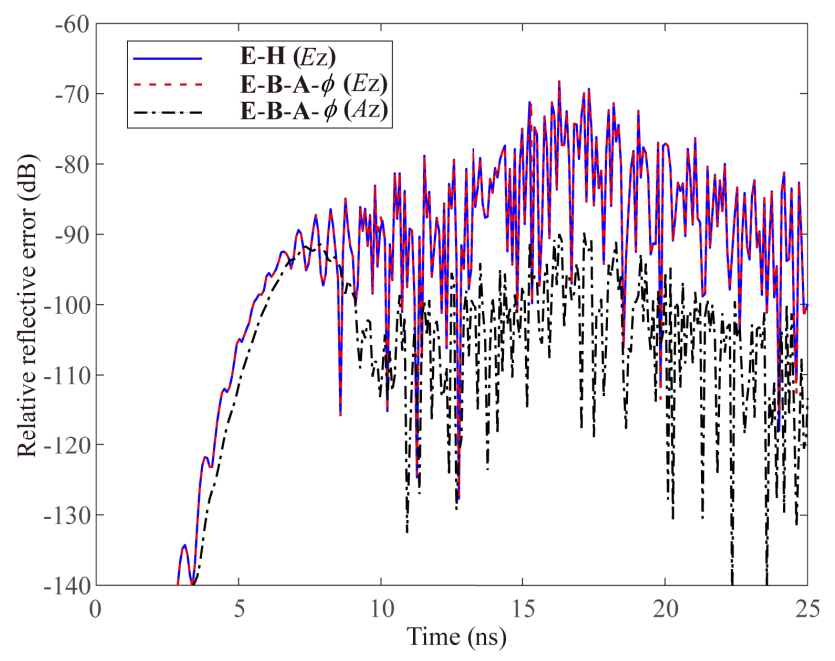

Fig. 10. Relative reflection errors of the probe point $E_{\mathrm{z}}$ for the $\mathbf{E}-\mathbf{B}-\mathbf{A}-\phi$ system and $\mathbf{E}-\mathbf{H}$ system, and the probe point $A_{\mathrm{z}}$ for the $\mathbf{E}-\mathbf{B}-\mathbf{A}-\phi$ system.

where $E(t)$ denotes the field component at the probe point and $E_{\text {ref }}(t)$ is the reference field value at the point within an enlarged simulation domain where there are no reflected EM waves.

As shown in Fig. 10, for the $E_{\mathrm{z}}$ field component at the probe point, both the proposed $\mathbf{E}-\mathbf{B}-\mathbf{A}-\phi$ numerical system and traditional $\mathbf{E}-\mathbf{H}$ numerical system have the comparable relative reflection error and the maximum value is around $-70 \mathrm{~dB}$. In addition, the relative reflection errors of the proposed $\mathbf{E}-\mathbf{B}-\mathbf{A}-\phi$ numerical system for the $A_{\mathrm{z}}$ field component at the probe point are also investigated, and the maximum value is around $-90 \mathrm{~dB}$. These results demonstrate the validation and efficiency of applying the CPML technique to the proposed system.

\section{MAXWELL-SCHRÖDINGER SYSTEM}

The progress of laser technology enables the control of quantum states of the electron. The problems of realizing the perfect switching of quantum states have attracted increasing interest over the past two decades. As mentioned in Section I, scientists have conducted extensive research on controlling the quantum states of a single electron by solving the semiclassical M-S equations with the FDTD solver. In addition, various forms of Maxwell's equations based on the $\mathbf{A}$ and $\phi$ formulas are derived and are to be coupled to the Schrödinger equation. The $\mathbf{A}$ and $\phi$ formulas introduced in the literature contain second-order partial differential operators, and thus, the corresponding simulation is usually time-consuming and complicated. Due to the simplicity of the proposed $\mathbf{E}-\mathbf{B}-\mathbf{A}-\phi$ equations, these EM equations are coupled to the following Schrödinger (29) and the quantum current density (30) to analyze the transient interaction between the incident EM field and the electron

$$
\begin{aligned}
i \hbar \frac{\partial}{\partial t} \psi(\mathbf{r}, t)= & -\frac{\hbar^{2}}{2 m_{0}} \nabla^{2}+\frac{i e \hbar}{m_{0}} \mathbf{A} \cdot \nabla+\frac{i e \hbar}{2 m_{0}}(\nabla \cdot \mathbf{A}) \\
& \left.+e \phi+V+\frac{e^{2}}{2 m_{0}} \mathbf{A}^{2}\right] \psi(\mathbf{r}, t) \\
\mathbf{J}(\mathbf{r}, t)= & \frac{-i e \hbar}{2 m_{0}}\left[\psi^{*}(\mathbf{r}, t) \nabla \psi(\mathbf{r}, t)-\psi(\mathbf{r}, t) \nabla \psi^{*}(\mathbf{r}, t)\right] \\
& -\frac{e^{2}}{m_{0}} \psi^{*}(\mathbf{r}, t) \mathbf{A} \psi(\mathbf{r}, t)
\end{aligned}
$$

where $\hbar$ is the reduced Planck's constant, $e$ and $m_{0}$ are the charge and mass of the electron, respectively, $V$ is the electrostatic quantum confinement potential, and $\psi$ is the wave function of the particle.

Meanwhile, the complex-valued calculations for solving (29) can be simplified to real-valued calculations via splitting the wave function into its real and imaginary parts

$$
\psi(\mathbf{r}, t)=\psi_{R}(\mathbf{r}, t)+i \psi_{I}(\mathbf{r}, t) .
$$

In addition, as for the model shown in Fig. 1, the timedependent wave function of this electron confined in a quasione-dimensional potential $V$ and subjected to a radiation field with $\phi$ and $A_{z}$.

Therefore, (29) can be derived as

$$
\begin{aligned}
& \frac{\partial}{\partial t} \psi_{I}(\mathbf{r}, t) \\
& =\frac{\hbar}{2 m_{0}} \nabla^{2} \psi_{R}(\mathbf{r}, t)+\frac{e}{m_{0}} \mathbf{A}_{z} \cdot \nabla \psi_{I}(\mathbf{r}, t) \\
& \quad+\frac{e}{2 m_{0}}\left(\nabla \cdot \mathbf{A}_{z}\right) \psi_{I}(\mathbf{r}, t)-\left(\frac{e \phi+V}{\hbar}+\frac{e^{2}}{2 m_{0} \hbar} \mathbf{A}_{z}^{2}\right) \psi_{R}(\mathbf{r}, t) \\
& \frac{\partial}{\partial t} \psi_{R}(\mathbf{r}, t) \\
& =-\frac{\hbar}{2 m_{0}} \nabla^{2} \psi_{I}(\mathbf{r}, t)+\frac{e}{m_{0}} \mathbf{A}_{z} \cdot \nabla \psi_{R}(\mathbf{r}, t) \\
& \quad+\frac{e}{2 m_{0}}\left(\nabla \cdot \mathbf{A}_{z}\right) \psi_{R}(\mathbf{r}, t)+\left(\frac{e \phi+V}{\hbar}+\frac{e^{2}}{2 m_{0} \hbar} \mathbf{A}_{z}^{2}\right) \psi_{I}(\mathbf{r}, t)
\end{aligned}
$$

and the polarization current (30) density is thus obtained

$$
\begin{aligned}
J_{z}(\mathbf{r}, t)=\frac{e \hbar}{2 m_{0}}\left[\psi_{R}(\mathbf{r}, t)\right. & \left.\nabla \psi_{I}(\mathbf{r}, t)-\psi_{I}(\mathbf{r}, t) \nabla \psi_{R}(\mathbf{r}, t)\right] \\
& -\frac{e^{2}}{m_{0}}\left(\psi_{R}^{2}(\mathbf{r}, t)+\psi_{I}^{2}(\mathbf{r}, t)\right) A_{z}
\end{aligned}
$$




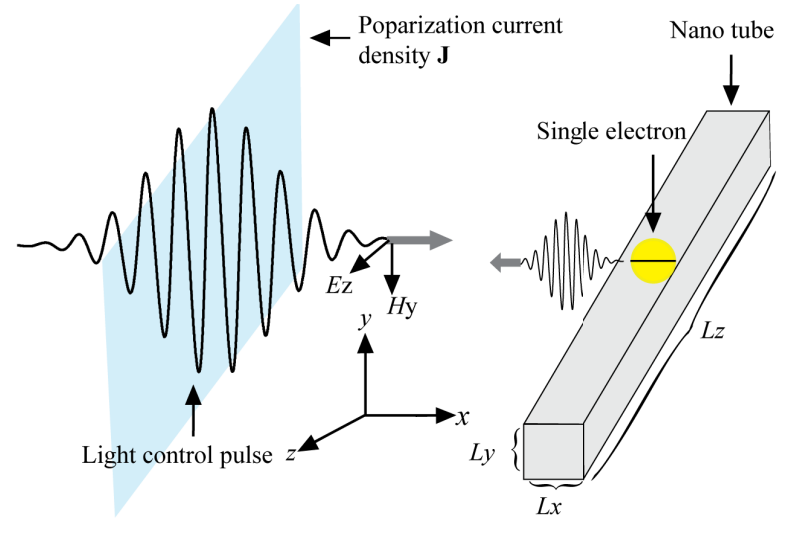

Fig. 11. Geometric model of a nanotube.

According to (32) and (33), we can see that the real and imaginary parts of wave function $\left(\psi_{I}(\mathbf{r}, t), \psi_{R}(\mathbf{r}, t)\right)$ appear at both sides of (32) and (33), respectively. Thus, the wave function should be sampled at the time steps of $n+1$ and $n+1 / 2$ when (32) and (33) are discretized as the discrete forms. Accordingly, two sets of iterative equations are constructed. The first group is used to solve the wave function at the time step of $n+1 / 2$, and the second group is used to solve the wave function at the time step of $n+1$, as shown in Fig. 1. The updating formulas for (32)-(34) are described in detail in the Appendix.

According to the theory of [20], the control pulse generator is designed to maximize the objective state $\psi_{1}$ of the electron from the ground state $\psi_{0}$. The control pulse $E^{s}$ can be generated by solving the following:

$$
E^{s}=-2 \frac{E_{0}}{m_{0}} \operatorname{Im}\left\langle\psi^{\prime}|W e z| \psi^{\prime}\right\rangle
$$

where $W$ represents the projection operator of $\left|\psi_{1}\right\rangle\left\langle\psi_{1}\right|$ and $\psi^{\prime}=e^{\left[-i(e / \hbar) A_{z}(z, t)\right]} \psi$ is a unitary transformation for the wave function $\psi$, and the derivation of (35) is introduced in [26]-[28]. At last, applying equations EM (6)-(8) and (11), Schrödinger (32) and (33), polarization current density (34), and the control pulse $E^{s}$ (35), the proposed scheme can provide a numerical simulation (as shown in Fig. 11), where a uniform plane-wave source excites the electrons in a nanotube to transform the quantum states of the electron from the ground state $\psi_{0}$ to the first excited state $\psi_{1}$.

Fig. 12 shows a procedure illustration of the proposed method for updating the field components in the coupled M-S system. First, the control pulse $E^{s}$ (35) is obtained by the wave function of the electron, and the polarization current density $J_{z}$ (34) generated by the electron motion will generate the radiation fields governed by the EM system [see (6)-(8) and (11)]. Then, the radiation fields will superimpose with the control pulse field $E^{s}$ as the total external EM field acting on the quantum system governed by (32) and (33), and thus, the steps of transforming the control pulse $E^{s}$ to $A^{s}$ and then superimpose with $A z$ are added in mulphysics simulation, as shown in Fig. 12. Although the incident EM field is polarized along the $z$-axis having thus only $E_{z}$ and $B_{y}$ components initially, the last field components are also needed

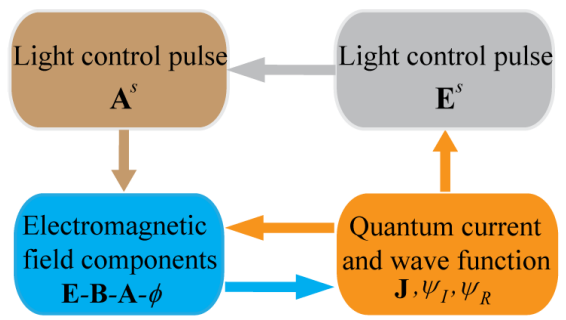

Fig. 12. Procedure illustration of the proposed method for updating the field components in the coupled $\mathrm{M}-\mathrm{S}$ system.

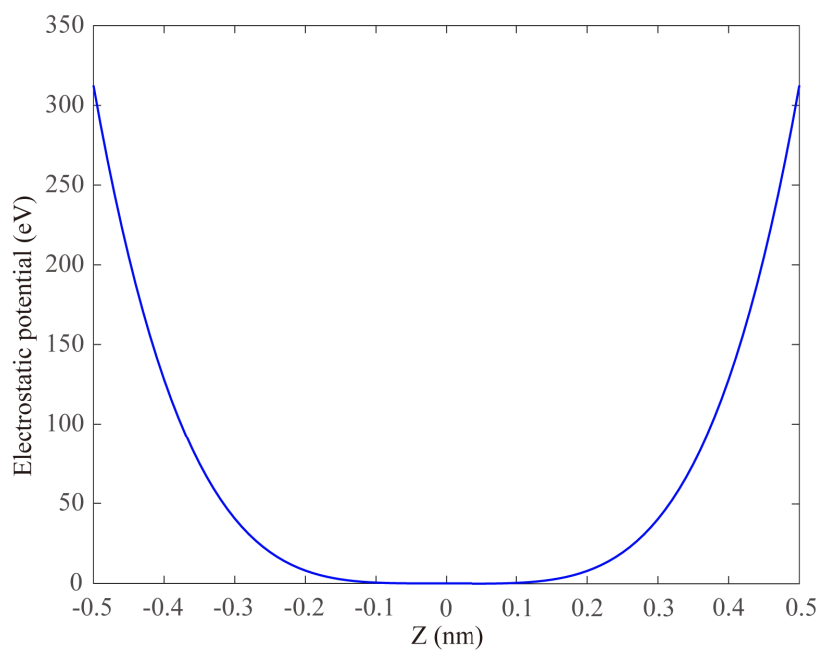

Fig. 13. Confining potential $V$ along the $z$-direction.

to interact with the electron. Therefore, all the fields, including $A_{x}, A_{y}, A_{z}, \phi, E_{x}, E_{y}, E_{z}, B_{x}, B_{y}$, and $B_{z}$, are updated in every iteration.

Finally, according to the program diagram of Fig. 12, the numerical multiphysics simulation is performed. For the EM system, the computational region contains $50 \times 50 \times 120$ Yee cells with the CPML region of ten layers, and the cell sizes are chosen as $\Delta x=\Delta y=1 \mathrm{~nm}$ and $\Delta z=0.01 \mathrm{~nm}$. For the QM system, as the electron is confined in the quasi-onedimensional space extending along the $z$-axis like a "tube," its wave function is dependent only on the $z$-coordinate. In other directions, the wave function is set to be uniform and the value is not zero only within one unit $\Delta x$ and $\Delta y$ of the Yee cell. Hence, the wave function is norm normalized as

$$
\Delta x \Delta y \int_{-\infty}^{\infty}|\psi(z)|^{2} d z=1
$$

and the length of the nanotube along the $z$-direction is chosen as $1 \mathrm{~nm}$; thus, 100 Yee cells are included in the QM system along the $z$-direction.

The ground state $\psi_{0}$ and the excited state $\psi_{1}$ with the confining potential $V$ (Fig. 13) are shown in Fig. 14. For numerical convergence, the wave function is initialized to be as

$$
\psi=\sqrt{0.9999} \psi_{0}+\sqrt{0.0001} \psi_{1} .
$$

As shown in Fig. 15, the electron wave packet $|\psi|^{2}$ initially exists as a single peak, which corresponds to the ground state 


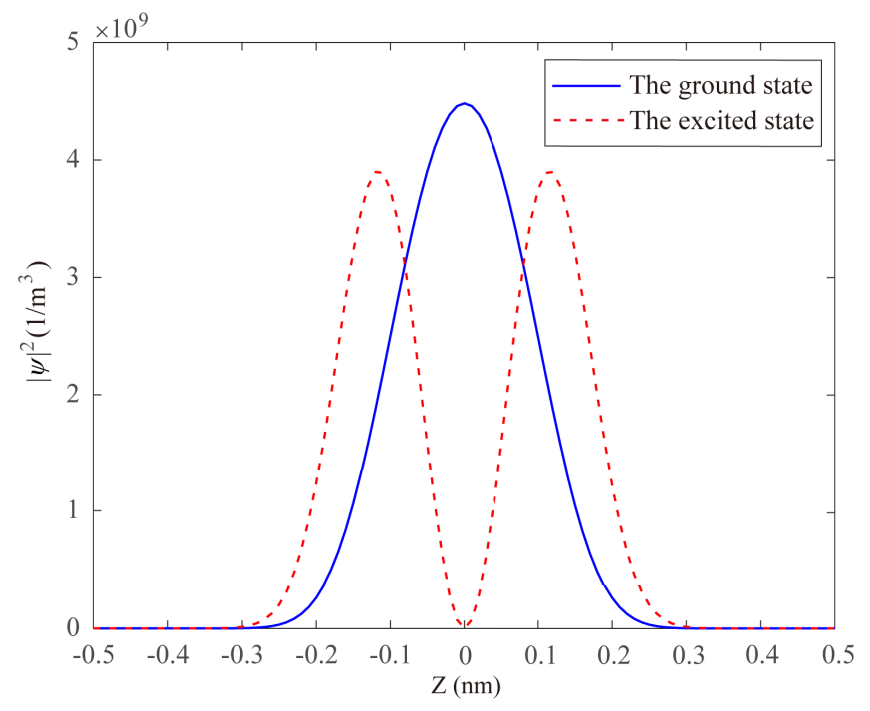

Fig. 14. Electron wave packet distribution of the ground state and the excited state. The corresponding eigenenergies are 2.05 and $7.35 \mathrm{eV}$, respectively.

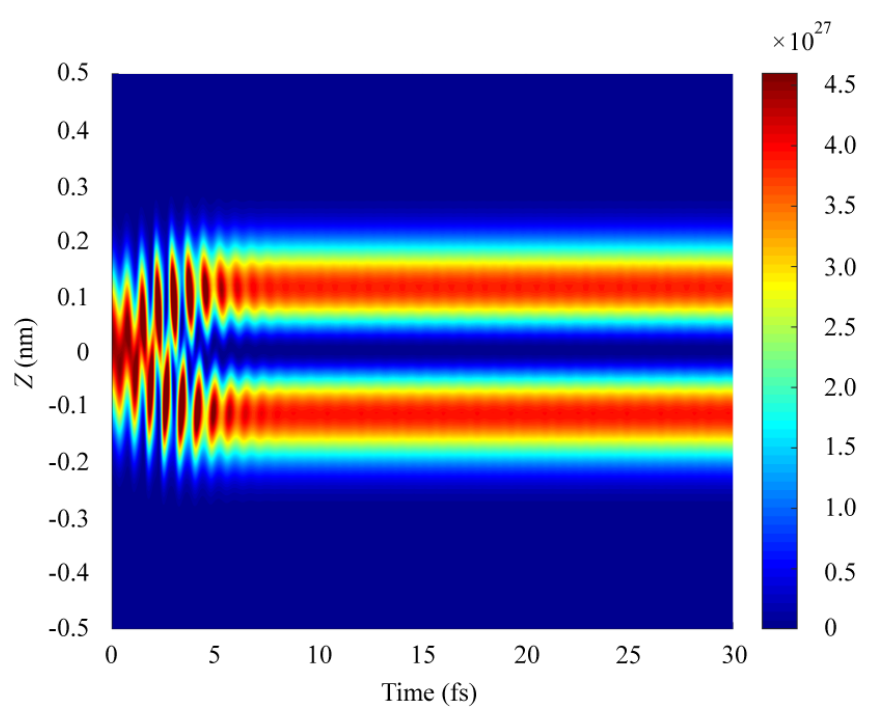

Fig. 15. Spatiotemporal plots of the probability density $|\psi|^{2}$.

of the electron, and then, the electron wave packet starts fluctuating about the time $2 \mathrm{fs}$. During the time interval of 2-10 fs, the electron wave packet oscillates rapidly under the illumination of the pulse and is finally converted into the bimodal form, which corresponds to the first excited state of the electron. In addition, to quantify the quantum state transition by the proposed scheme, the transition rate factor $\Omega_{k}$ is defined as

$$
\Omega_{k}=\left\langle\psi^{\prime} \mid \psi_{k}\right\rangle\left\langle\psi_{k} \mid \psi^{\prime}\right\rangle
$$

where $\psi_{k}$ denotes the quantum state of the electron. The resultant variations of the factors are shown in Fig. 16. As the simulation time increases, the factor $\Omega_{0}$ decreases from one to zero and the factor $\Omega_{1}$ increases from zero to one when the pulse gradually raises $(t=3-10 \mathrm{fs})$. Finally, the value of factor $\Omega_{1}$ remains stable when the pulse has gone. It can

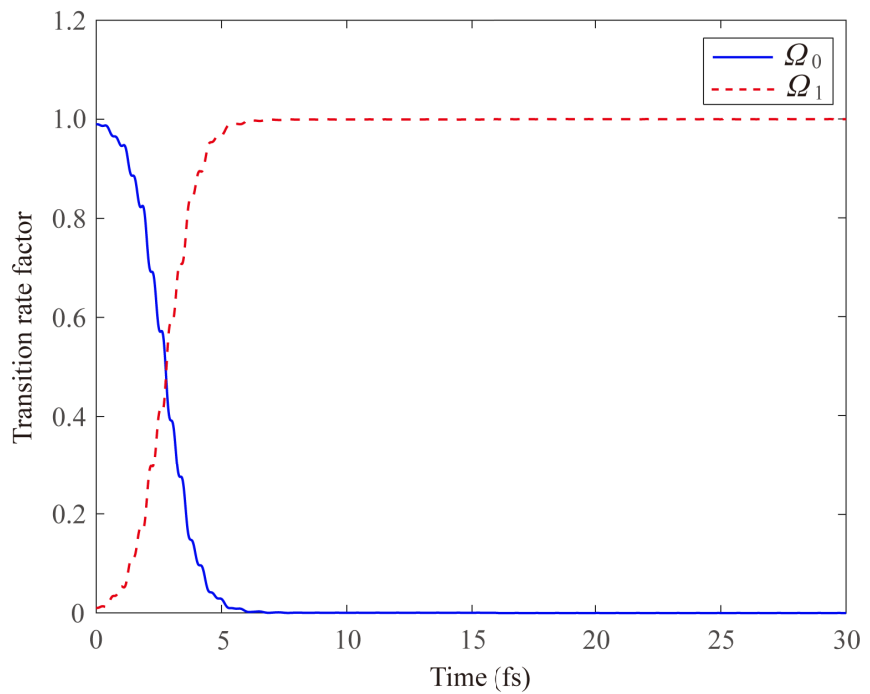

Fig. 16. Magnitude of the transition rate factor $\Omega$.

be concluded that the perfect quantum state transition is numerically achieved by using the proposed $\mathrm{M}-\mathrm{S}$ system.

\section{CONCLUSiON}

Universal vector-scalar potentials system is proposed to model the EM response from arbitrary inhomogeneous media and demonstrate the gauge invariance. The numerical results show that both simple gauge and generalized Lorenz gauge can be adopted to calculate the time evolution of EM fields in dispersive and nondispersive inhomogeneous media, and the results obtained by the two gauges are nearly identical. In view of simplicity and stability, the simple gauge is suggested to be used. Moreover, the method of applying the CPML technique to the proposed system is introduced, and the numerical results demonstrate the validation and efficiency of the CPML technique.

In addition, the proposed method provides a convenient way to calculate the vector and scalar potentials, which is essential to model the semiclassical quantum EM problems described by the $\mathrm{M}-\mathrm{S}$ equations. The combination of the proposed EM system with the Schrödinger equation is presented to design laser pulse for controlling discrete quantum states. The simulation results show that the proposed method reproduces a perfect quantum state switching while with a more straightforward numerical solution process. In future, the proposed numerical method will be used to manipulate the discrete multiquantum states and other multiphysics problems in more complex environments where irregular structures are included.

\section{APPENDIX}

For the EM part, according to the spatial and temporal configuration of the field components as shown in Fig. 1, the symbols $i, j$, and $k$ denote spatial coordinates, while $n$ denotes the sampling point in time domain. Then, according to the time-evolution procedure of the $\mathbf{E}-\mathbf{B}-\mathbf{A}-\phi$ framework as shown in Fig. 2, the updating equations for the 
EM framework are expressed as (39)-(44), shown at the bottom of the page, where $m$ denotes $(i+(1 / 2), j, k)$, $(i, j+(1 / 2), k)$, and $(i, j, k+(1 / 2))$, respectively, and

$$
\begin{aligned}
& C A(m)=\left(1-\frac{\sigma(m) \Delta t}{2 \varepsilon(m)}\right) /\left(1+\frac{\sigma(m) \Delta t}{2 \varepsilon(m)}\right) \\
& C B(m)=\frac{\Delta t}{\varepsilon(m)} /\left(1+\frac{\sigma(m) \Delta t}{2 \varepsilon(m)}\right) .
\end{aligned}
$$

For the scalar potential $\mathbf{A}$ and $\phi$, we have

$$
\begin{aligned}
& A_{x}^{n+\frac{1}{2}}\left(i+\frac{1}{2}, j, k\right) \\
&= A_{x}^{n-\frac{1}{2}}\left(i+\frac{1}{2}, j, k\right)-\Delta t E_{x}^{n}\left(i+\frac{1}{2}, j, k\right) \\
& \quad-\frac{\Delta t}{\Delta x}\left[\phi^{n}(i+1, j, k)-\phi^{n}(i, j, k)\right] \\
& A_{y}^{n+\frac{1}{2}}\left(i, j+\frac{1}{2}, k\right) \\
&=A_{y}^{n-\frac{1}{2}}\left(i, j+\frac{1}{2}, k\right)-\Delta t E_{y}^{n}\left(i, j+\frac{1}{2}, k\right) \\
& \quad-\frac{\Delta t}{\Delta y}\left[\phi^{n}(i, j+1, k)-\phi^{n}(i, j, k)\right]
\end{aligned}
$$

$$
\begin{gathered}
A_{z}^{n+\frac{1}{2}}\left(i, j, k+\frac{1}{2}\right) \\
=A_{z}^{n-\frac{1}{2}}\left(i, j, k+\frac{1}{2}\right)-\Delta t E_{z}^{n}\left(i, j, k+\frac{1}{2}\right) \\
-\frac{\Delta t}{\Delta z}\left[\phi^{n}(i, j, k+1)-\phi^{n}(i, j, k)\right] \\
\phi^{n+1}(i, j, k) \\
=\phi^{n}(i, j, k)-\frac{\Delta t}{\mu_{0} \varepsilon_{\phi}^{2}} \\
\times\left\{\frac { 1 } { \Delta x } \varepsilon _ { A _ { x } } ( i + \frac { 1 } { 2 } , j , k ) \left[A_{x}^{n+\frac{1}{2}}\left(i+\frac{1}{2}, j, k\right)\right.\right. \\
\left.\quad-A_{x}^{n+\frac{1}{2}}\left(i-\frac{1}{2}, j, k\right)\right] \\
+\frac{1}{\Delta y} \varepsilon_{A_{y}}\left(i, j+\frac{1}{2}, k\right)\left[A_{y}^{n+\frac{1}{2}}\left(i, j+\frac{1}{2}, k\right)\right. \\
\left.\quad-A_{y}^{n+\frac{1}{2}}\left(i, j-\frac{1}{2}, k\right)\right] \\
\quad-\frac{1}{\Delta z} \varepsilon_{A_{z}}\left(i, j, k+\frac{1}{2}\right)\left[A_{z}^{n+\frac{1}{2}}\left(i, j, k+\frac{1}{2}\right)\right. \\
\left.\left.\quad-A_{z}^{n+\frac{1}{2}}\left(i, j, k-\frac{1}{2}\right)\right]\right\} .
\end{gathered}
$$

$$
\begin{aligned}
& B_{x}^{n+\frac{1}{2}}\left(i, j+\frac{1}{2}, k+\frac{1}{2}\right)=\frac{1}{\Delta y}\left(A_{z}^{n+\frac{1}{2}}\left(i, j+1, k+\frac{1}{2}\right)-A_{z}^{n+\frac{1}{2}}\left(i, j, k+\frac{1}{2}\right)\right) \\
& -\frac{1}{\Delta z}\left(A_{y}^{n+\frac{1}{2}}\left(i, j+\frac{1}{2}, k+1\right)-A_{y}^{n+\frac{1}{2}}\left(i, j+\frac{1}{2}, k\right)\right) \\
& B_{y}^{n+\frac{1}{2}}\left(i+\frac{1}{2}, j, k+\frac{1}{2}\right)=\frac{1}{\Delta z}\left(A_{x}^{n+\frac{1}{2}}\left(i+\frac{1}{2}, j, k+1\right)-A_{x}^{n+\frac{1}{2}}\left(i+\frac{1}{2}, j, k\right)\right) \\
& -\frac{1}{\Delta x}\left(A_{z}^{n+\frac{1}{2}}\left(i+1, j, k+\frac{1}{2}\right)-A_{z}^{n+\frac{1}{2}}\left(i, j, k+\frac{1}{2}\right)\right) \\
& B_{z}^{n+\frac{1}{2}}\left(i+\frac{1}{2}, j+\frac{1}{2}, k\right)=\frac{1}{\Delta x}\left(A_{y}^{n+\frac{1}{2}}\left(i+1, j+\frac{1}{2}, k\right)-A_{y}^{n+\frac{1}{2}}\left(i, j+\frac{1}{2}, k\right)\right) \\
& -\frac{1}{\Delta y}\left(A_{x}^{n+\frac{1}{2}}\left(i+\frac{1}{2}, j+1, k\right)-A_{x}^{n+\frac{1}{2}}\left(i+\frac{1}{2}, j, k\right)\right) \\
& E_{x}^{n+1}\left(i+\frac{1}{2}, j, k\right)=C A(m) E_{x}^{n}\left(i+\frac{1}{2}, j, k\right)+C B(m) \\
& \times\left[\begin{array}{l}
\left(B_{z}^{n+1 / 2}\left(i+\frac{1}{2}, j+\frac{1}{2}, k\right)-B_{z}^{n+1 / 2}\left(i+\frac{1}{2}, j-\frac{1}{2}, k\right)\right) / \Delta y \\
-\left(B_{y}^{n+1 / 2}\left(i+\frac{1}{2}, j, k+\frac{1}{2}\right)-B_{z}^{n+1 / 2}\left(i+\frac{1}{2}, j, k-\frac{1}{2}\right)\right) / \Delta z
\end{array}\right] \\
& E_{y}^{n+1}\left(i, j+\frac{1}{2}, k\right)=C A(m) \cdot E_{y}^{n+1}\left(i, j+\frac{1}{2}, k\right)+C B(m) \\
& \times\left[\begin{array}{l}
\left(B_{x}^{n+1 / 2}\left(i, j+\frac{1}{2}, k+\frac{1}{2}\right)-B_{x}^{n+1 / 2}\left(i, j+\frac{1}{2}, k-\frac{1}{2}\right)\right) / \Delta z \\
-\left(B_{z}^{n+1 / 2}\left(i+\frac{1}{2}, j+\frac{1}{2}, k\right)-B_{z}^{n+1 / 2}\left(i-\frac{1}{2}, j+\frac{1}{2}, k\right)\right) / \Delta x
\end{array}\right] \\
& E_{z}^{n+1}\left(i, j, k+\frac{1}{2}\right)=C A(m) \cdot E_{z}^{n+1}\left(i, j, k+\frac{1}{2}\right)+C B(m) \\
& \times\left[\begin{array}{l}
\left(B_{y}^{n+1 / 2}\left(i+\frac{1}{2}, j, k+\frac{1}{2}\right)-B_{y}^{n+1 / 2}\left(i-\frac{1}{2}, j, k+\frac{1}{2}\right)\right) / \Delta x \\
-\left(B_{x}^{n+1 / 2}\left(i, j+\frac{1}{2}, k+\frac{1}{2}\right)-B_{x}^{n+1 / 2}\left(i, j-\frac{1}{2}, k+\frac{1}{2}\right)\right) / \Delta y
\end{array}\right]
\end{aligned}
$$


For the QM part, the updating equations for the discrete forms of (32)-(34) are expressed as

$$
\begin{aligned}
\psi_{R}^{n+\frac{1}{2}}(k) & \\
= & \psi_{R}^{n-\frac{1}{2}}(k)-\frac{\Delta t \hbar}{2 m_{0}} \alpha\left[\psi_{I}^{n}(k)\right] \\
& +\frac{\Delta t e}{m_{0}} A_{z}^{n}(k) \beta\left[\psi_{R}^{n}(k)\right]+\frac{\Delta t e}{2 m_{0}} \beta\left[A_{z}^{n}(k)\right] \psi_{R}^{n}(k) \\
& +\left(\frac{\Delta t e^{2}}{2 m_{0} \hbar}\left(A_{z}^{n}(k)\right)^{2}+\frac{\Delta t V(k)}{\hbar}+\frac{\Delta t e \phi^{n}(k)}{\hbar}\right) \psi_{I}^{n}(k) \\
\psi_{I}^{n+} & \frac{1}{2}(k) \\
= & \psi_{I}^{n-\frac{1}{2}}(k)+\frac{\Delta t \hbar}{2 m_{0}} \alpha\left[\psi_{R}^{n}(k)\right] \\
& +\frac{\Delta t e}{m_{0}} A_{z}^{n}(k) \beta\left[\psi_{I}^{n}(k)\right]+\frac{\Delta t e}{2 m_{0}} \beta\left[A_{z}^{n}(k)\right] \psi_{I}^{n}(k) \\
& -\left(\frac{\Delta t e^{2}}{2 m_{0} \hbar}\left(A_{z}^{n}(k)\right)^{2}+\frac{\Delta t V(k)}{\hbar}+\frac{\Delta t e \phi^{n}(k)}{\hbar}\right) \psi_{R}^{n}(k) \\
\psi_{R}^{n+1} & (k) \\
= & \psi_{R}^{n}(k)-\frac{\Delta t \hbar}{2 m_{0}} \alpha\left[\psi_{I}^{n+\frac{1}{2}}(k)\right] \\
& +\frac{\Delta t e}{m_{0}} A_{z}^{n+\frac{1}{2}}(k) \beta\left[\psi_{R}^{n+\frac{1}{2}}(k)\right]+\frac{\Delta t e}{2 m_{0}} \beta\left[A_{z}^{n+\frac{1}{2}}(k)\right] \psi_{R}^{n+\frac{1}{2}}(k) \\
& \left(\frac{\Delta t e^{2}}{2 m_{0} \hbar}\left(A_{z}^{n+\frac{1}{2}}(k)\right)^{2}+\frac{\Delta t V(k)}{\hbar}+\frac{\Delta t e \phi^{n+\frac{1}{2}}(k)}{\hbar}\right) \psi_{I}^{n+\frac{1}{2}}(k)
\end{aligned}
$$

$$
\begin{aligned}
\psi_{I}^{n+1}(k) & \\
= & \psi_{I}^{n}(k)+\frac{\Delta t \hbar}{2 m_{0}} \alpha\left[\psi_{R}^{n+\frac{1}{2}}(k)\right] \\
& +\frac{\Delta t e}{m_{0}} A_{z}^{n+\frac{1}{2}}(k) \beta\left[\psi_{I}^{n+\frac{1}{2}}(k)\right]+\frac{\Delta t e}{2 m_{0}} \beta\left[A_{z}^{n+\frac{1}{2}}(k)\right] \psi_{I}^{n+\frac{1}{2}}(k) \\
& -\left(\frac{\Delta t e^{2}}{2 m_{0} \hbar}\left(A_{z}^{n+\frac{1}{2}}(k)\right)^{2}+\frac{\Delta t V(k)}{\hbar}+\frac{\Delta t e \phi^{n+\frac{1}{2}}(k)}{\hbar}\right) \psi_{R}^{n+\frac{1}{2}}(k) .
\end{aligned}
$$

After the imaginary and real parts of wave function are calculated by (51)-(54), the polarization current $J_{z}^{n+(1 / 2)}$ is obtained by

$$
\begin{aligned}
J_{z}^{n+1}(k) & \\
= & \frac{e \hbar}{m_{0}}\left\{\psi_{R}^{n+\frac{1}{2}}(k) \beta\left[\psi_{I}^{n+\frac{1}{2}}(k)\right]-\psi_{I}^{n+\frac{1}{2}}(k) \beta\left[\psi_{R}^{n+\frac{1}{2}}(k)\right]\right\}-\frac{e^{2}}{2 m_{0}} \\
\times & {\left[\left(\psi_{R}^{n+\frac{1}{2}}(k)\right)^{2}+\left(\psi_{I}^{n+\frac{1}{2}}(k)\right)^{2}\right] } \\
\times & {\left[A_{z}^{n+\frac{1}{2}}\left(i, j, k+\frac{1}{2}\right)+A_{z}^{n+\frac{1}{2}}\left(i, j, k-\frac{1}{2}\right)\right] . }
\end{aligned}
$$

The operators $\alpha$ and $\beta$ represent the sixth-order accurate difference formulas of the first- and second-order derivatives with respect to the $z$-coordinate $\left(\partial / \partial k\right.$ and $\left.\partial^{2} / \partial k^{2}\right)$, respectively. For an arbitrary function $\chi$, the sixth-order accurate difference formulas of the first- and second-order derivatives are expressed as

$$
\begin{aligned}
& \beta[\chi(k)]=\frac{1}{60 \Delta z}\left[\begin{array}{l}
\chi(k+3)-9 \chi(k+2)+45 \chi(k+1) \\
-45 \chi(k-1)+9 \chi(k-2)-\chi(k-3)
\end{array}\right] \\
& \alpha[\chi(k)]=\frac{1}{90 \Delta z^{2}}\left[\begin{array}{l}
\chi(k+3)-13.5 \chi(k+2)+135 \chi(k+1) \\
-245 \chi(k)+135 \chi(k-1) \\
-13.5 \chi(k-2)+\chi(k-3)
\end{array}\right] .
\end{aligned}
$$

The stability condition of the QM system that is simulated by the FDTD simulation has been investigated in [29] and is defined as

$$
\Delta t \leq \frac{2 \hbar}{\frac{2 \hbar^{2}}{m_{0}}\left(\frac{1}{\Delta x^{2}}+\frac{1}{\Delta y^{2}}+\frac{1}{\Delta z^{2}}\right)+V_{\max }}
$$

where $V_{\max }$ is the maximum value. In order to have a stable scheme, the time step $\Delta t$ should be taken to the minimum of the EM or QM systems. In the next, the numerical stability of the proposed $\mathbf{E}-\mathbf{B}-\mathbf{A}-\phi$ system is analyzed and provided as follows.

According to the updating equations of the EM fields and the auxiliary variables (39)-(50), the matrix form of the updating equation can be represented by (all the field components are set at the same time for simplicity)

$$
\left[M_{l}\right] P^{n+1}=\left[M_{r}\right] P^{n}
$$

where

$$
P=\left(E_{x}, E_{y}, E_{z}, B_{x}, B_{y}, B_{z}, A_{x}, A_{y}, A_{z}, \phi\right) .
$$

$M_{l}$ and $M_{r}$ are expressed by (61) and (62), respectively

$$
\begin{aligned}
M_{l}=\left[\begin{array}{cccccccccc}
a & 0 & 0 & 0 & 0 & 0 & 0 & 0 & 0 & 0 \\
0 & a & 0 & 0 & 0 & 0 & 0 & 0 & 0 & 0 \\
0 & 0 & a & 0 & 0 & 0 & 0 & 0 & 0 & 0 \\
0 & 0 & 0 & 1 & 0 & 0 & 0 & 0 & 0 & 0 \\
0 & 0 & 0 & 0 & 1 & 0 & 0 & 0 & 0 & 0 \\
0 & 0 & 0 & 0 & 0 & 1 & 0 & 0 & 0 & 0 \\
1 & 0 & 0 & 0 & 0 & 0 & b & 0 & 0 & 0 \\
0 & 1 & 0 & 0 & 0 & 0 & 0 & b & 0 & 0 \\
0 & 0 & 1 & 0 & 0 & 0 & 0 & 0 & b & 0 \\
0 & 0 & 0 & 0 & 0 & 0 & D_{x} & D_{y} & D_{z} & c
\end{array}\right] \\
M_{r}=\left[\begin{array}{ccccccccccc}
a & 0 & 0 & 0 & D_{z} & -D_{y} & 0 & 0 & 0 & 0 \\
0 & a & 0 & -D_{z} & 0 & D_{x} & 0 & 0 & 0 & 0 \\
0 & 0 & a & D_{y} & -D_{z} & 0 & 0 & 0 & 0 & 0 \\
0 & 0 & 0 & 1 & 0 & 0 & 0 & -D_{z} & D_{y} & 0 \\
0 & 0 & 0 & 0 & 1 & 0 & D_{z} & 0 & -D_{x} & 0 \\
0 & 0 & 0 & 0 & 0 & 1 & -D_{y} & D_{x} & 0 & 0 \\
1 & 0 & 0 & 0 & 0 & 0 & b & 0 & 0 & D_{x} \\
0 & 1 & 0 & 0 & 0 & 0 & 0 & b & 0 & D_{y} \\
0 & 0 & 1 & 0 & 0 & 0 & 0 & 0 & b & D_{z} \\
0 & 0 & 0 & 0 & 0 & 0 & 0 & 0 & 0 & c
\end{array}\right]
\end{aligned}
$$

where $a=-\mu \varepsilon / \Delta t, b=-1 / \Delta t, c=-\mu \varepsilon_{\phi}^{2} /\left(\Delta t \varepsilon_{A}\right)$, and $D_{u}=\partial / \partial u(u=x, y, z)$ denotes the first-order derivative of $u$ versus space. 
We assume that the expression of the field components $P$ in space domain can be described by

$$
P_{i, j, k}^{n}=\phi_{P} \zeta^{n} e^{-j\left(k_{x} \Delta x+k_{y} \Delta y+k_{z} \Delta z\right)}
$$

where $\zeta$ is the growth factor and $k_{u}(u=x, y, z)$ is the wavenumber. Therefore, the spatial derivative of the field components $P_{i, j, k}^{n}$ can be approximated as

$$
D_{u} P_{i, j, k}^{n}=\delta_{u} \phi_{P} \zeta^{n} e^{-j\left(k_{x} \Delta x+k_{y} \Delta y+k_{z} \Delta z\right)}
$$

and $\delta_{u}=2 j \sin \left(k_{u} \Delta u / 2\right) / \Delta u(u=x, y, z)$.

Substituting (63) and (64) into (59) gives

$$
\left[M_{l} \zeta-M_{r}\right] P^{n}=M P^{n}=0
$$

and

$$
\begin{aligned}
& \text { M } \\
& =\left[\begin{array}{cccccccccc}
a \zeta_{1} & 0 & 0 & 0 & -D_{z} & D_{y} & 0 & 0 & 0 & 0 \\
0 & a \zeta_{1} & 0 & D_{z} & 0 & -D_{x} & 0 & 0 & 0 & 0 \\
0 & 0 & a \zeta_{1} & -D_{y} & D_{x} & 0 & 0 & 0 & 0 & 0 \\
0 & 0 & 0 & \zeta_{1} & 0 & 0 & 0 & D_{z} & -D_{y} & 0 \\
0 & 0 & 0 & 0 & \zeta_{1} & 0 & -D_{z} & 0 & D_{x} & 0 \\
0 & 0 & 0 & 0 & 0 & \zeta_{1} & D_{y} & -D_{x} & 0 & 0 \\
1 & 0 & 0 & 0 & 0 & 0 & b \zeta_{1} & 0 & 0 & -D_{x} \\
0 & 1 & 0 & 0 & 0 & 0 & 0 & b \zeta_{1} & 0 & -D_{y} \\
0 & 0 & 1 & 0 & 0 & 0 & 0 & 0 & b \zeta_{1} & -D_{z} \\
0 & 0 & 0 & 0 & 0 & 0 & D_{x} \zeta & D_{y} \zeta & D_{z} \zeta & c \zeta_{1}
\end{array}\right]
\end{aligned}
$$

where $\zeta_{1}=\zeta-1$.

To determine the nonzero solution of (66), the determinant of the coefficient matrix $M$ in (66) should be zero. The polynomial of the determinant of the coefficient matrix $M$ is obtained through many simplified operations and expressed as

$$
\begin{aligned}
a \zeta^{3}(\zeta-1)\left[\left(\sigma_{x}^{2}+\right.\right. & \left.\left.\sigma_{y}^{2}+\sigma_{z}^{2}\right) \zeta-b c(\zeta-1)^{2}\right] \\
& \times\left[\left(\sigma_{x}^{2}+\sigma_{y}^{2}+\sigma_{z}^{2}\right) \zeta-a b(\zeta-1)^{2}\right]^{2} .
\end{aligned}
$$

According to the von Neuman stability analysis method, the module value of the growth factor $\zeta$ should be no more than 1. For the first polynomial factor of (67), the growth factors are $\zeta_{1,2,3}=0$, and for the second polynomial factor of (67), the growth factor is $\zeta_{4}=1$, thereby meeting the numerical stability requirement.

For the third polynomial factor of (67), the growth factor $\zeta$ is obtained by

$\zeta_{5,6}=\frac{-\left(\sigma_{x}^{2}+\sigma_{x}^{2}+\sigma_{x}^{2}+2 b c\right) \pm \sqrt{\left(\sigma_{x}^{2}+\sigma_{x}^{2}+\sigma_{x}^{2}+2 b c\right)-4 b^{2} c^{2}}}{-2 b c}$.

As mentioned above, the module value of the growth factor $\zeta_{5,6}$ in (68) should be no more than 1 . Then, the following condition should be satisfied:

$$
4 b^{2} c^{2} \geq\left(\sigma_{x}^{2}+\sigma_{x}^{2}+\sigma_{x}^{2}+2 b c\right) .
$$

Substituting $\delta_{u}=2 j \sin \left(k_{u} \Delta u / 2\right) / \Delta u(u=x, y, z)$ into (69), thus,

$\left[\frac{\sin \left(k_{x} \Delta x / 2\right)}{\Delta x / 2}\right]^{2}+\left[\frac{\sin \left(k_{x} \Delta y / 2\right)}{\Delta y / 2}\right]^{2}+\left[\frac{\sin \left(k_{x} \Delta z / 2\right)}{\Delta z / 2}\right]^{2} \leq 4 b^{2} c^{2}$.

Since $\sin \left(k_{u} \Delta u / 2\right)$ can be at most one, then we get

$$
\left[\frac{1}{\Delta x / 2}\right]^{2}+\left[\frac{1}{\Delta y / 2}\right]^{2}+\left[\frac{1}{\Delta z / 2}\right]^{2} \leq 4 b^{2} c^{2} .
$$

As $b=-1 / \Delta t$ and $c=-\mu \varepsilon_{\phi}^{2} /\left(\Delta t \varepsilon_{A}\right)$, we get

$$
b c=\mu \varepsilon_{\phi}^{2} /\left(\Delta t^{2} \varepsilon_{A}\right) .
$$

Substituting (72) into (71), we get

$$
\Delta t=\sqrt{\mu \varepsilon_{\phi}^{2} / \varepsilon_{A}} \frac{1}{\sqrt{(1 / \Delta x)^{2}+(1 / \Delta y)^{2}+(1 / \Delta z)^{2}}} .
$$

Although the values of permittivity $\varepsilon_{A}$ and $\varepsilon_{\phi}$ are related to the spatial location of $\mathbf{A}$ and $\phi$, respectively, they are equal to the same value $\varepsilon$. Then, the inequality of time step $\Delta t$ is derived as

$$
\Delta t=\sqrt{\mu \varepsilon} \frac{1}{\sqrt{(1 / \Delta x)^{2}+(1 / \Delta y)^{2}+(1 / \Delta z)^{2}}} .
$$

For the fourth polynomial factor of (67), the growth factor $\zeta$ is obtained by

$$
\begin{aligned}
\zeta_{7,8} & =\zeta_{9,10} \\
& =\frac{-\left(\sigma_{x}^{2}+\sigma_{x}^{2}+\sigma_{x}^{2}+2 a b\right) \pm \sqrt{\left(\sigma_{x}^{2}+\sigma_{x}^{2}+\sigma_{x}^{2}+2 b c\right)-4 a^{2} b^{2}}}{-2 a b} .
\end{aligned}
$$

Similarly, according to (75), we can get the following:

$$
\left[\frac{1}{\Delta x / 2}\right]^{2}+\left[\frac{1}{\Delta y / 2}\right]^{2}+\left[\frac{1}{\Delta z / 2}\right]^{2} \leq 4 a^{2} b^{2} .
$$

As $a=-\mu \varepsilon / \Delta t$ and $b=-1 / \Delta t$, we get

$$
a b=\mu \varepsilon /\left(\Delta t^{2}\right) .
$$

Substituting (77) into (76), then the inequality of the time step $\Delta t$ is derived as

$$
\Delta t=\sqrt{\mu \varepsilon} \frac{1}{\sqrt{(1 / \Delta x)^{2}+(1 / \Delta y)^{2}+(1 / \Delta z)^{2}}} .
$$

According to (74) and (78), we can conclude that the proposed E-B-A- $\phi$ system satisfies the Courant-FriedrichLevy (CFL) limitation of the conventional FDTD method.

In conclusion, to have a stable scheme, the time step $\Delta t$ should be taken to the minimum of the EM system (58) or QM system (78). 


\section{REFERENCES}

[1] J.-M. Jin, Theory and Computation of Electromagnetic Fields. Hoboken, NJ, USA: Wiley, 2010.

[2] A. A. Omar and Z. Shen, "A compact and wideband vertically polarized monopole antenna," IEEE Trans. Antennas Propag., vol. 67, no. 1, pp. 626-631, Jan. 2019.

[3] K. Belli, C. M. Rappaport, H. Zhan, and S. Wadia-Fascetti, "Effectiveness of 2-D and 2.5-D FDTD ground-penetrating radar modeling for bridge-deck deterioration evaluated by 3-D FDTD," IEEE Trans. Geosci. Remote Sens., vol. 47, no. 11, pp. 3656-3663, Nov. 2009.

[4] J. Yan et al., "VCSEL-based in-circuit status-monitoring and effectsdiagnosis method for HPEM susceptibility test on digital electronic equipment," IEEE Trans. Electromagn. Compat., vol. 60, no. 1, pp. 234-242, Feb. 2018.

[5] J. D. Griffiths, Introduction to Quantum Mechanics. Cambridge U.K.: Cambridge Univ. Press, 2016.

[6] A. Capua, O. Karni, and G. Eisenstein, "A finite-difference time-domain model for quantum-dot lasers and amplifiers in the Maxwell-Schrödinger framework," IEEE J. Sel. Topics Quantum Electron., vol. 19, no. 5, Sep./Oct. 2013, Art. no. 1900410.

[7] R. Fleischhaker and J. Evers, "A Maxwell-Schrödinger solver for quantum optical few-level systems," Comput. Phys. Commun., vol. 182, pp. 739-747, Oct. 2010.

[8] K. Lopata and D. Neuhauser, "Multiscale Maxwell-Schrödinger modeling: A split field finite-difference time-domain approach to molecular nanopolaritonics," J. Chem. Phys., vol. 130, 2009, Art. no. 104707.

[9] K. Lopata and D. Neuhauser, "Nonlinear nanopolaritonics: Finitedifference time-domain Maxwell-Schrödinger simulation of moleculeassisted plasmon transfer," J. Chem. Phys., vol. 131, 2009, Art. no. 014701

[10] J. W. You and N. C. Panoiu, "Analysis of the interaction between classical and quantum plasmons via FDTD-TDDFT method," IEEE J. Multiscale Multiphys. Comput. Techn., vol. 4, pp. 111-118, 2019.

[11] H. Takeda and S. John, "Self-consistent Maxwell-Bloch theory of quantum-dot-population switching in photonic crystals," Phys. Rev. A: Gen. Phys., vol. 83, no. 5, May 2011, Art. no. 053811.

[12] G. Alagappan, "Slow scale Maxwell-Bloch equations for active photonic crystals," Prog. Electromagn. Res. B., vol. 55, pp. 169-194, 2013.

[13] G. Demeter, "Solving the Maxwell-Bloch equations for resonant nonlinear optics using GPUs," Comput. Phys. Commun., vol. 184, pp. 1203-1210, Apr. 2013.

[14] C. Jayun Ryu, A. Y. Liu, W. E. I. Sha, and W. Cho Chew, "Finitedifference time-domain simulation of the Maxwell-Schrödinger system," IEEE J. Multiscale Multiphys. Comput. Techn., vol. 1, pp. 40-47, 2016.

[15] X. Chen, F. M. Kong, K. Li, and M. Liu, "A high-order symplectic FDTD scheme for the Maxwell-Schrodinger system," IEEE J. Quantum Electron., vol. 54, no. 1, Feb. 2018, Art. no. 6100108.

[16] L. Pierantoni, D. Mencarelli, and T. Rozzi, "A new 3-D transmission line matrix scheme for the combined Schrödinger-Maxwell problem in the electronic/electromagnetic characterization of nanodevices," IEEE Trans. Microw. Theory Techn., vol. 56, no. 3, pp. 654-662, Mar. 2008.

[17] A. Taflove and S. C. Hagness, Computational Electrodynamics the Finite difference Time-Domain Method, 3rd ed. Boston, MA, USA: Artech House, 2005.

[18] I. Ahmed, E. H. Khoo, E. P. Li, and R. Mittra, "A hybrid approach for solving coupled Maxwell and Schrödinger equations arising in the simulation of nano-devices," IEEE Antennas Wireless Propag. Lett., vol. 9, pp. 914-916, 2010.

[19] I. Ahmed and E. Li, "An hybrid FDTD and ADI-FDTD technique for coupled Maxwell's and Schrodinger's equations," in Proc. IEEE Antennas Propag. Soc. Int. Symp. (APSURSI), Jul. 2010, pp. 1-4.

[20] T. Takeuchi, S. Ohnuki, and T. Sako, "Maxwell-Schrödinger hybrid simulation for optically controlling quantum states: A scheme for designing control pulses," Phys. Rev. A: Gen. Phys., vol. 91, Mar. 2015, Art. no. 033401

[21] Y. P. Chen, W. E. I. Sha, L. Jiang, M. Meng, Y. M. Wu, and W. C. Chew, "A unified Hamiltonian solution to Maxwell-Schrödinger equations for modeling electromagnetic field-particle interaction," Comput. Phys. Commun., vol. 215, pp. 63-70, Jun. 2017.

[22] G. D. Xie, Z. X. Huang, M. Fang, and W. E. I. Sha, "Simulating Maxwell-Schrödinger equations by high-order symplectic FDTD algorithm," IEEE J. Multiscale Multiphys. Comput. Techn., vol. 4, pp. 143-151, 2019.

[23] W. C. Chew, "Vector potential electromagnetics with generalized gauge for inhomogeneous media: Formulation," Prog. Electromagn. Res., vol. 149, pp. 69-84, 2014.
[24] J. A. Roden and S. D. Gedney, "Convolution PML (CPML): An efficient FDTD implementation of the CFS-PML for arbitrary media," Microw. Opt. Technol. Lett., vol. 27, no. 5, pp. 334-339, Dec. 2000.

[25] J. Li, H. Jiang, X. Zhao, and N. Feng, "Effective CNAD- and ADE-based CFS-PML formulations for truncating the dispersive FDTD domains,' IEEE Antennas Wireless Propag. Lett., vol. 14, pp. 1267-1270, 2015.

[26] C. Xiang, F. Kong, K. Li, and M. Liu, "A quantum state controller based on the electromagnetic potentials," IEEE Access, vol. 7, pp. 42006-42013, 2019.

[27] A. Q. Cheng, H. Zeng, S. T. Chen, and R. S. Chen, "d'Alembert-Schrödinger hybrid simulation for laser-induced multiquantum state transitions in a three-dimensional artificial atom," Opt Lett., vol. 44, no. 17, pp. 4399-4402, Feb. 2019.

[28] Y. Ohtsuki, H. Kono, and Y. Fujimura, "Quantum control of nuclear wave packets by locally designed optimal pulses," J. Chem. Phys., vol. 109, no. 21, pp. 9318-9331, Dec. 1998.

[29] P. B. Visscher, "A fast explicit algorithm for the time-dependent Schrödinger equation," Comput. Phys., vol. 5, no. 6, pp. 596-597, 1991.

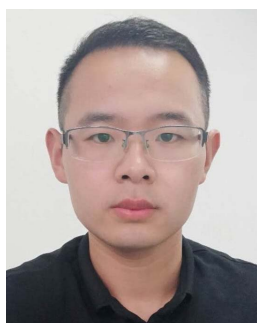

Guoda Xie was born in Anhui, China, in 1991. He received the M.S. and Ph.D. degrees from the School of Electronics and Information Engineering, Anhui University, Hefei, China, in 2017 and 2020, respectively.

From September 2019 to July 2020, he was a Ph.D. exchange student with Zhejiang University, Hangzhou, China. His research interests include computational electromagnetics, unconditionally stable FDTD method, symplectic FDTD method, and multiphysics modeling with time-domain numerical methods.

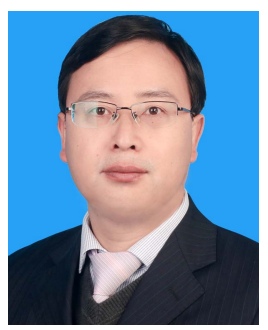

Zhixiang Huang (Senior Member, IEEE) was born in Anhui, China, in 1979. He received the B.S. and Ph.D. degrees from Anhui University (AHU), Hefei, China, in 2002 and 2007, respectively.

Since 2008, he has been a Full Professor with the School of Electronic Information and Engineering, AHU. From September 2010 to 2011, he was a Visiting Scholar with Iowa State University, Ames, IA, USA. From August 2013 to October 2013, he was a Visiting Professor with the University of Hong Kong, Hong Kong. From February 2014 to February 2015, he was a Visiting Professor with the Beijing National Laboratory for Condensed Matter Physics, Institute of Physics, Chinese Academy of Sciences, Beijing, China. He has coauthored one monograph on the symplectic finite-difference time-domain method and two book chapters for CRC Press and InTech Publishers. He has coauthored 90 peer-reviewed journal articles included in the Web of Science Core Collection. His current research interests include time-domain numerical methods, metamaterials, and active metamaterials.

Prof. Huang was a recipient of the Second Prize of Science and Technology from the Anhui Government, China, in 2015, and the National Science Foundation for Outstanding Young Scholar of China, in 2017.

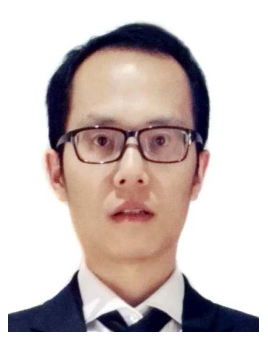

Jian Wei You (Senior Member, IEEE) received the B.Sc. degree in electrical engineering from Xidian University, Xi'an, China, in 2010, and the Ph.D. degree in electromagnetic field and microwave techniques from Southeast University, Nanjing, China, in 2016.

From January 2016 to December 2020, he was a Research Associate with the Department of Electronic and Electrical Engineering, University College London, London, U.K. In March 2021, he joined Southeast University, Nanjing, China, as a Research Professor. His research interests include computational electromagnetic algorithm, microwave and millimeter-wave circuits and antennas simulations, multiphysics and multiscale simulation, plasmonics, topological photonics, and nonlinear optics and quantum information. 


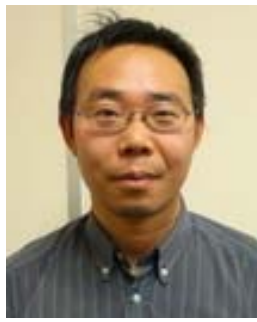

Zhihao Lan received the B.Sc. and M.Sc. degrees from Beijing Institute of Technology, and Peking University, Beijing, China, in 2004 and 2007, respectively, and the Ph.D. degree from Heriot-Watt University, Edinburgh, U.K., in 2012, all in physics.

He worked as a Post-Doctoral Research Fellow with Southampton University, Southampton, U.K., from 2011 to 2014, and Nottingham University, Nottingham, U.K., from 2014 to 2017. He joined the Department of Electronic and Electrical Engineering, University College London, London, U.K., in 2018, as a Research Associate in theoretical and computational modeling of quantum photonic materials and devices. His research interests include quantum optics, quantum gases, quantum many-body physics, quantum nonequilibrium systems, novel quantum systems for quantum simulations and quantum technologies, topological states of matter, topological photonics, and plasmonics.

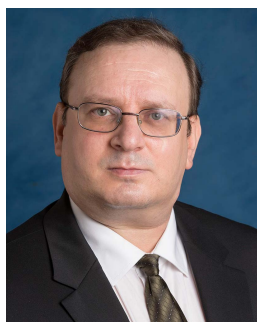

Nicolae C. Panoiu (Member, IEEE) received the B.Sc. and M.S. degrees in physics from the University of Bucharest, Bucharest, Roma, in 1990 and 1992, respectively, and the Ph.D. degree from New York University, New York, NY, USA, in 2001.

After graduating from NYU, he was a PostDoctoral Fellow with the Department of Applied Physics and Applied Mathematics, Columbia University, New York. He is currently Professor of Nanophotonics with the Department of Electronic and Electrical Engineering, University College London, London, U.K. His research interests include silicon photonics, optical properties of photonic nanostructures and metamaterials, and computational modeling of electromagnetic structures.

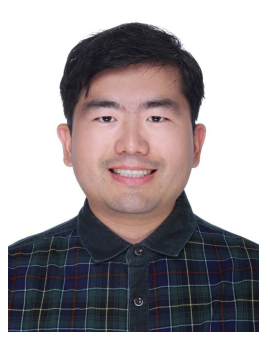

Wei E. I. Sha (Senior Member, IEEE) received the B.S. and Ph.D. degrees in electronic engineering from Anhui University, Hefei, China, in 2003 and 2008, respectively.

From July 2008 to 2017, he was a Post-Doctoral Research Fellow and then a Research Assistant Professor with the Department of Electrical and Electronic Engineering, University of Hong Kong, Hong Kong. From March 2018 to 2019, he worked with the University College London, London, U.K., as a Marie Skłodowska-Curie Individual Fellow. From October 2017, he joined the College of Information Science and Electronic Engineering with Zhejiang University, Hangzhou, China, where he is currently a tenure-track Assistant Professor. His research interests include theoretical and computational research in electromagnetics and optics, focusing on the multiphysics and interdisciplinary research. He has authored or coauthored 150 refereed journal articles, 135 conference publications (including 38 invited talks), eight book chapters, and two books. His Google Scholar citation is 6500 with an H-index of 39. His research involves fundamental and applied aspects in computational and applied electromagnetics, nonlinear and quantum electromagnetics, micro- and nano-optics, optoelectronic device simulation, and multiphysics modeling.

Dr. Sha is a member of OSA. He served as a reviewer for 60 technical journals and a technical program committee member of nine IEEE conferences. He also served as an Associate Editor for IEEE JOURNAL ON Multiscale AND Multiphysics Computational TeChNiques, IEEE Open Journal of ANTENNAS AND PROPAGation, and IEEE ACCESS. In 2015, he was awarded Second Prize of Science and Technology from Anhui Government, Hefei, China. In 2007, he was awarded the Thousand Talents Program for Distinguished Young Scholars of China. He received six Best Student Paper Prizes and one Young Scientist Award with his students. 